\title{
Karakterisation af oversættelse for de til Chomsky hierarkiet hørende automater
}

Erik Meineche Schmidt

DAIMI PB - 3

Marts 1972

DATALOGISK AFDELING AARHUS UNIVERSITET

Ny Munkegade, Bygn. 540 8000 Aarhus C, Denmark

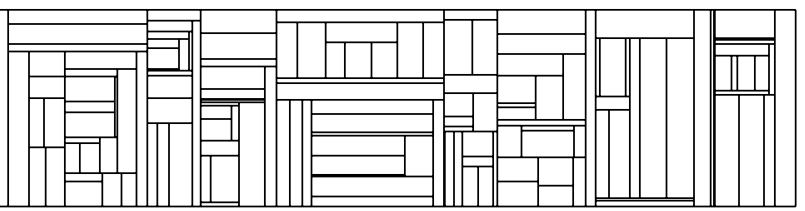


Karakterisation af oversættelse for de til

Chomsky-hierarkiet h $\phi r e n d e$ automater

Erik Meineche Schmidt

1972 


\section{INDLEDNING}

Nærværende skrift behandler f $\phi l$ gende problemstilling:

Givet en af de til Chomsky-hierarkiet hфrende automater, f. eks. en push-down acceptor. Forsyn denne med output, dvs. sфrg for at hver gang automaten 'foretager' sig noget, giver det anledning til at der på en envejs output tape skrives en streng over et alfabet. Definer grafen for en sådan maskine som mængden af par $(x, y)$, hvor input $x$ dels tilhorer det sprog, automaten accepterer, dels giver anledning til output y. Hvorledes kan sådanne grafer karakteriseres for de forskellige typer af automater?

Historisk set starter behandlingen af den emnekreds, der er beslægtet med ovenstående, med Ginsburg og Rose [1966], som indfфrer begrebet transducere, dvs. automater uden sluttilstande, der anvendes som en slags generaliserede afbildninger på sprog.

Lewis og Stearns [1968] beskæftiger sig med oversættelse af såvel deterministiske som ikke-deterministiske context-free sprog, Aho og Ullıman [1969a] indf $\phi r e r$ begrebet syntax-dirigeret oversættelsesskema for context-free sprog, medens Ibarra [1971] er den forste til at anvende de af Ginsburg og Greibach [1969] introducerede Familier af: Abstrakte Sprog og Automater. I en helt ny artikel behandler Culik II og Morey [ 197 1] bl. a. for forste gang oversættelse defineret af linezrt begrænsede automater.

Den her givne fremstilling skal tjene to formål. Dels som speciale i forbindelse med hovedfagseksamen i datalogi og dels som materiale i forbindelse med et 2. dels kursus i Formelle Sprog og Automater. Der er derfor her og der medtaget beviser, som er "overflфdige"l i den forstand, at de $i$ forvejen findes $i$ litteraturen. 
INDHOLD

Side I : Indledning

Afsnit 1: Notation og lignende

Afsnit 2: Abstrakt familie af sprog og abstrakt familie af automater

Afsnit 3: Abstrakt familie af oversættere

Afsnit 4: Lineært begrænsede automater og lineært begrænsede oversættere

Afsnit 5: Turing acceptorer og Turing oversættere

Afsnit 6: Konklusion og afslutning 


\section{AFSNIT 1}

\section{NOTATION OG LIGNENDE}

Vi vil i vid udstrækning anvende samme notation som Hopcroft og Ulliman [1969].

Således vil vi - med mindre andet udtrykkeligt er angivet - altid have at

store bogstaver betegner hjælpesymboler

små bogstaver i begyndelsen af alfabetet betegner terminale symboler

små bogstaver i slutningen af alfabetet betegner strenge af terminale symboler

små græske bogstaver betegner strenge bestående af såvel hjælpe- som terminale symboler.

Et alfabet er altid en endelig ikke tom mangde af symboler. Hvis $\Sigma$ er et alfabet, er $\Sigma^{*}$ som sædvanlig mængden af strenge over

$\Sigma$. Hvis vi betegner det tomme ord med $\epsilon$ er $\Sigma^{+}=\Sigma^{*} \backslash\{\epsilon\}$.

Hvis $D$ er en mangde, betyder $|D|$ antallet af elementer $i D$ og $\rho_{\text {fin }}(D)$ betyder mængden af endelige delmængder af $D$.

Hvis $\alpha$ er en streng, er $|\alpha|$ antallet af elementer $i$ strengen.

Lad $\Sigma, \Delta$ være to alfabeter. En homomorfi $h$ er en afbildning $\mathrm{h}: \Sigma^{*} \rightarrow \Delta^{*}$ som opfylder

$$
\begin{gathered}
h(\epsilon)=\epsilon \\
\forall x, y \in \sum^{*}: h(x y)=h(x) h(y)
\end{gathered}
$$

En grammatik er et system $G=\left(V_{N}, V_{T}, P, S\right)$ hvor $V_{N}$ er hjælpesymboler, $V_{T}$ er terminale symboler, med $V=V_{N} \cup V_{T}$ er $P$ produktioner af formen $\alpha \rightarrow \beta, \alpha \in V^{+}, \beta \in V^{*}$, og endelig er $s \in V_{N}$ startsymbolet. Relationen $\Rightarrow$ mellem strenge $\mathbf{i} V^{*}$ de- 
fineres ved at $\alpha \Rightarrow \beta$, hvis der findes $\mu, \delta \in V^{*}$ således at $\alpha=\mu \omega \delta, \beta=\mu \eta \delta$ og $\omega \rightarrow \eta$ er en produktion. Betegner vi med $\stackrel{*}{\Rightarrow}$ den transitive, refleksive lukning af $\Rightarrow$, er sproget genereret af $G$ mængden $L(G)=\left\{w \in V_{T}^{*} \mid S \stackrel{*}{\Rightarrow} w\right\}$.

En grammatik som den ovenfor definerede er altid en Turing grammatik.

Lad $G$ vare en Turing grammatik. Safremt der for enhver produktion $\alpha \rightarrow \beta$ gælder at $|\beta| \geq|\alpha|$ er $G$ context sensitiv.

Lad $G$ vare en Turing grammatik. Såfremt der for enhver produktion $\alpha \rightarrow \beta$ gælder at $\alpha \in V_{N}$ er $G$ context-free.

Lad $G$ være en context-free grammatik. Såfremt det for enhver produktion $A \rightarrow \beta$ gælder at $\beta \in\left(V_{T} \cup\{\varepsilon\}\right) \cdot\left(V_{N} \cup\{\varepsilon\}\right)$, er $G$ regulær.

Bemærk, at det iflg. ovenstående er umuligt for et context sensitivt sprog at indeholde det tomme ord. Dette kommer man normalt ud over ved at tillade produktionen $S \rightarrow \epsilon$ forudsat at $S$ ikke optiæder på hфjresiden af nogen produktion. Af tekniske årsager vil vi imidlertid her og $i$ det f $\phi$ lgende fastholde den oprindelige definition.

Klasserne af sprog horende til de fire typer af grammatikker vil vi betegne med hhv. RE, CS, CF og REG.

Da vi senere får brug for Post Correspondence Problem (PCP), vil $v i$ indfore dette her:

Lad $\Sigma$ være et alfabet med $|\Sigma| \geq 2$ og lad $A=\left\{x_{1}, \ldots, x_{k}\right\}$, $B=\left\{w_{1}, \ldots, w_{k}\right\}$ være lister bestående af $k$ strenge $x_{i}, w_{i} \in \Sigma^{+}, 1 \leq i \leq k$. Sådanne lister vil vi kalde PCP-lister. 
Vi siger, at PCP med lister $A$ og $B$ har en $\mid \phi$ sning, såfremt der findes heltal $i_{1}, \ldots, i_{\text {hy }}, m \geq 1$ med $i_{j} \leq k, 1 \leq j \leq m$ således at $x_{i_{1}} \ldots x_{i_{m}}=w_{i_{1}} \ldots w_{i_{m}}$. I så fald skriver vi $\operatorname{PCP}(A, B)=$ 1 og ellers $P C P(A, B) \stackrel{1}{=} 0$. Det er velkendt, at $P C P$ er rekursivt ul $\phi$ seligt.

Lad os slutte dette afsnit med at anfфre, at vi overalt $i$ fremstillingen vil bruge symbolet $\square$ til at afslutte sætninger, lemmaer og corrollarer samt beviser herfor. 
ABSTRAKT FAMILIE AF SPROG OG ABSTRAKT FAMILIE AF AUTOMATER

Vi har folgende definition fra Ginsburg og Greibach [1969]:

Definition 2.1 En Abstrakt Familie af sprog (AFL) er et par $(\Sigma, \mathscr{L})$ eller $\mathscr{L}$ hvis $\Sigma$ er underforståt hvor

i) $\quad \Sigma$ er en uendelig mangde af symboler

ii) $\forall L \in \mathscr{L}$ 马 $\Sigma_{I} \subseteq \Sigma\left|\Sigma_{I}\right|<\infty: L \subseteq \Sigma_{i}^{*}$

iii) $\mathscr{L}$ er lukket under operationerne $U, \cdot,+, \epsilon_{\text {-free ho- }}$ momorfi, invers homomorfi og snit med regulære mængder

iv) $\exists L \in \mathscr{L}: L \neq \varnothing$

Definition 2.2 En AFL $\mathscr{L}$ siges at være full, såfremt $\mathscr{L}$ er lukket under vilkårlig homomorfi.

Folgende lemma er bevist overalt i litteraturen. Vi viser det alligevel her, fordi vi senere vil gфre kraftigt brug af det.

Lemma 2.3 Lad $L \in R E$. Der findes da et $L_{I} \in C S$ og en homomorfi $h$ således at $h\left(L_{1}\right)=L$.

Bevis $L a d G=\left(V_{N}, V_{T}, P, S\right)$ være en grammatik med $L(G)=L$ og lad \# vare et symbol, som ikke er med $i V_{N} \cup V_{T}$. $V i$ kan uden indskrænkning antage, at alle produktioner i $P$ er på formen

$$
\begin{array}{ll}
\alpha \rightarrow \beta & \alpha, \beta \in V_{N}^{*} \\
A \rightarrow a & A \in V_{N}, a \in V_{T}
\end{array}
$$

Betragt grammatikken $G_{1}=\left(V_{N}, V_{T} \cup\{\#\}, P_{I}, S\right)$, som har $f \phi \mid-$ gende produktioner 


$$
\begin{aligned}
& \text { i) } \alpha \rightarrow \beta \in P_{I} \text { hvis } \alpha \rightarrow \beta \in P \wedge|\beta| \geq|\alpha| \\
& \text { ii) } \alpha \rightarrow \beta \#^{k} \in P_{I} \text { hvis }\left\{\begin{array}{l}
\alpha \rightarrow \beta \in P \wedge|\beta|<|\alpha| \\
k=|\alpha|-|\beta|
\end{array}\right. \\
& \text { iii) } A \rightarrow A \# \text { for alle } A \in V_{N}
\end{aligned}
$$

Definer $h$ ved

$$
h(a)= \begin{cases}a \text { hvis } & a \in V_{T} \\ \varepsilon- & a=\#\end{cases}
$$

Det er klart, at $h\left(L_{1}\right)=L$

Betragtes Chomsky-hierarkiet, er det velkendt, at hver af klasserne REG, CF, RE er en full AFL, medens CS kun er en AFL.

Næste skridt er at knytte en familie af automater til en familie af sprog. Vi har f $\phi l g e n d e$ definition fra Ginsburg og Greibach [1969].

Definition 2.4 En Abstrakt Familie af (envejs non-deterministiske) Acceptorer (AFA) er et par $(\Omega, D)$ eller $D$ hvis $\Omega$ er underforståt med f $\phi$ lgende egenskaber

i) $\Omega$ er et system (K, $\Sigma, I, 1, f, g)$ hvor

a) $K, \Sigma$ er uendelige mængder og $\Gamma$, 1 er mængder med $\Gamma \neq \phi, 1 \neq \varnothing$

b) $f$ er en afbildning $f: \Gamma^{*} \times 1 \rightarrow \Gamma^{*} \cup\{\phi\}$

c) $g$ er en afbildning $g: \Gamma^{*} \rightarrow P_{\text {fin }}\left(\Gamma^{*}\right)$ således at $g(\epsilon)=\epsilon$ og $\epsilon \in g(\gamma)$ hvis og kun hvis $\gamma=\varepsilon$

d) for ethvert $\gamma \in g\left(\Gamma^{*}\right)$ findes der $1 \gamma \in 1$ så $f\left(\gamma^{1}, 1 \gamma\right)=\gamma^{\prime}$ for alle $\gamma^{\prime}$ med $\gamma \in g\left(Y^{\prime}\right)$

e) for ethvert $u \in I$ findes der en endelig mængde $\Gamma_{u} \subseteq \Gamma$ saledes at hvis $\Gamma_{I} \subseteq \Gamma, \gamma \in \Gamma_{I}^{*}$ og $f(\gamma, u) \neq \varnothing$ så er $f(\gamma, u) \in\left(I_{I} \cup I_{u}\right)^{*}$ 
2.3

ii) D er familien af elementer (acceptorer) af formen $D=\left(K_{1}, \Sigma_{1}, \delta, q_{0}, F\right)$ hvor

a) $K_{1}, \Sigma_{1}$ er endelige delmangder af $K, \Sigma_{0} F \subseteq K_{1}$, $q_{0} \in K_{1}$

b) $\delta$ er en afbildning

$\delta: K_{I} \times\left(\Sigma_{I} \cup\{\varepsilon\}\right) \times g\left(\Gamma^{*}\right) \rightarrow P_{f i n}\left(K_{I} \times I\right)$

således at mængden

$G_{D}=\left\{\gamma \mid \exists q \in K_{I}, \exists a \in \Sigma_{I} U\{\varepsilon\}: \delta(q, a, \gamma) \neq \varnothing\right\}$ er endelig.

Definition 2.5 Lad $(\Omega, D)$ være en AFA og lad $D=\left(K_{I}, \Sigma_{I}, \delta, q_{0}, F\right)$ være en acceptor. - er en relation på $K_{I} \times \Sigma_{I}^{*} \times \Gamma^{*}$ defineret ved:

For $a \in \Sigma_{1} U\{\epsilon\}$ er $(q, a x, \gamma) H\left(p, x, y^{\prime}\right)$ såfremt der findes $\bar{\gamma}$ og u med $\bar{\gamma} \in g(\gamma),(p, u) \in \delta(q, a, \bar{\gamma})$ og $f(\gamma, u)=\gamma^{\prime}$.

Lad $H^{*}$ være den reflexive, transitive lukning af - . Ved sproget accepteret af $D$ vil vi forstå mangden

$$
L(D)=\left\{w \in \Sigma_{I} * \mid \exists p \in F:\left(q_{0}, w, \varepsilon\right) H_{*}(p, \epsilon, \varepsilon)\right\}
$$

$V$ i definerer også $\mathscr{L}(D)=\{L(D) \mid D \in D\}$

Eksempler på hvorledes nogle af de sædvanlige typer af automater falder inden for denne definition, findes $i$ Ginsburg og Greibach [1969]. Det er også her, vi finder hovedsætningen om sammenhængen mellem AFL og AFA, nemlig

Sætning 2.6 Lad $\mathcal{F}$ være en AFL. Der findes da en AFA $D$ således at $\mathcal{F}=\mathscr{L}(\mathcal{D})$ hvis og kun hvis $F$ er en full AFL.

Heraf folger umiddelbart, at der til familierne REG, CF, RE findes AFA'er med de $\mathrm{i}$ sætning 2.6 næunte egenskaber. Disse AFAler er familierne af endelige automater, push down automater og Turing maskiner. 
En oversætter (translator) er $\mathrm{i}$ denne sammenhang en acceptor, der er udstyret med en output-tape og et dertil horende output-alfabet. Hver gang maskinen "foretager sig noget", giver den som output et ord over output-alfabetet.

Eksempel 3.1 Lad $M=\left(K, \Sigma, \Gamma, \delta, q_{0}, Z_{0}, F\right)$ vare en pushdown automat som defineret i Hopcroft og Ullmann [1969]. Transitionsfunktionen $\delta$ er en afbildning

$$
\delta: K \times(\Sigma U\{\epsilon\}) \times \Gamma \rightarrow P_{f i n}\left(K \times \Gamma^{*}\right)
$$

En push-down translator $M^{\prime}=\left(K, \Sigma, \Gamma, \Delta, \delta 1, q_{0}, Z_{0}, F\right)$ fås ved at tilf $\phi$ je output-alfabetet $\Delta$ og udvide billedmængden for $\delta$ til

$$
\delta 1: K \times(\Sigma U\{\epsilon\}) \times \Gamma \rightarrow \mathcal{P}_{\text {fin }}\left(K \times \Gamma^{*} \times \Delta *\right)
$$

Dette generaliseres i f $\phi$ Igende definitioner fra lbarra [1971].

Definition 3.2 En abstrakt familie af (envejs non-deterministiske) oversættere (AFTR) er et par $(\Omega, M)$ eller $\mathcal{M}$ hvis $\Omega$ er underforstået hvor

i) $\Omega$ er et system (K, $\Sigma, I$, I, f, g) med samme egenskaber som i def. 2. 4 .

ii) $M$ er familien af elementer (oversættere) $M=\left(K_{1}, \Sigma_{1}, \Sigma_{2}, \delta, q_{0}, F\right)$ hvor

a) $K_{1}, \Sigma_{1}, q_{0}, F$ er som $i$ def. 2.4

b) $\Sigma_{2} \subseteq \Sigma \quad$ med $0<\left|\Sigma_{2}\right|<\infty$

c) $\delta$ er en afbildning

$\delta: K_{1} \times\left(\Sigma_{1} \cup\{\epsilon\}\right) \times g\left(\Gamma^{*}\right) \rightarrow P_{f i n}\left(K_{1} \times 1 \times \Sigma_{2}^{*}\right)$

således at mangden

$G_{M}:\left\{\gamma \mid \exists a \in \Sigma_{1} \cup\{\varepsilon\}, \exists q \in K_{1}: \quad \delta(q, a, \gamma) \neq \phi\right\}$ er endelig. 
Definition 3.3 Lad M være en AFTR og lad $M=\left(K_{1}, \Sigma_{1}, \Sigma_{2}, \delta, q_{0}, F\right)$ være en oversætter $i \mathcal{M} . \mid-$ er en relation på $K_{1} \times \Sigma_{1}^{*} \times \Gamma^{*} \times \Sigma_{2}^{*}$ defineret ved

$$
\begin{aligned}
& \text { for } a \in \Sigma_{I} \cup\{\epsilon\} \text { er }(q, a \times, \gamma, y) \mid-\left(p, x, \gamma^{\prime}, y v\right) \text { såfremt } \\
& \text { der findes } \bar{Y} \text { og } u \text { med } \bar{Y} \in g(\gamma)(p, u, v) \in \delta(q, a, \bar{\gamma}) \text { og } \\
& f(\gamma, u)=\gamma^{\prime}
\end{aligned}
$$

Lad $H^{*}$ være den ref lexive, transitive lukning af $H_{\text {. Ved grafen }}$ for $M$ vil vi forstå mængden

$$
\operatorname{Gr}(M)=\left\{(x, y)\left|\exists p \in F:\left(q_{o}, x, \epsilon, \epsilon\right)\right|^{*}(p, \epsilon, \epsilon, y)\right\}
$$

ogvi sætter $\operatorname{Gr}(M)=\{G r(M) \mid M \in M\}$

Definition 3.4 Lad $M$ være en oversætter. Ved definitionsmængden for $M$ vil vi forstå mængden

$$
\operatorname{Dom}(M)=\{x \mid(x, y) \in \operatorname{Gr}(M)\}
$$

og ved billedmængden

$$
\operatorname{Ran}(M)=\{y \mid(x, y) \in G r(M)\}
$$

Definition 3.5 Lad $(\Omega, M)$ være en AFTR og lad $\left(\Omega_{1}, 9\right)$ være en AFA. $M$ og $D$ siges at være associerede såfremt $\Omega=\Omega_{1}$.

Hvis $M$ er en AFTR, har vi folgende sætning fra Ibarra [1971], der karakteriserer $\mathrm{Gr}(\mathcal{M})$.

Sætning 3.6 Lad $\mathcal{M}$ være en AFTR og lad $\mathcal{D}$ være dens associerede AFA. Lad $\Sigma_{2}, \Sigma_{3}$ være endelige delmængder af $\Sigma$, og lad $T \subseteq \Sigma_{2} * \times \Sigma_{3} *$. Der gælder da, at der findes $M \in \mathcal{M}$, således at $T=\operatorname{Gr}(M)$ hvis og kun hvis der findes en endelig mængde $\Sigma_{q} \subseteq \Sigma$, et sprog $L \in \mathscr{L}(\mathcal{D}), L \subseteq \Sigma_{1} *$ og to homomorfier $h_{1}: \Sigma_{1} * \rightarrow \Sigma_{2} *$, $h_{2}: \Sigma_{1} * \rightarrow \Sigma_{3}^{*}$ således at

$$
T=\left\{\left(h_{1}(w), h_{2}(w)\right) \mid w \in L\right\} .
$$




\section{Bevis}

a) Lad $T \subseteq \Sigma_{2}^{*} \times \Sigma_{3} *$ og lad $M_{1}=\left\{K_{1}, \Sigma_{2}, \Sigma_{3}, \delta, q_{0}, F\right\}$ være i $M$ med $T=\operatorname{Gr}\left(M_{1}\right)$.

Srt $n=\max \left\{|y| \mid(p, u, y) \in \delta_{1}(q, a, \gamma),(q, a, \gamma) \in\right.$ $\left.K_{1} \times\left(\Sigma_{2} \cup\{\epsilon\}\right) \times G_{M_{1}}\right\}$. At $n$ eksisterer, f $\phi$ lger af def. 3.2 ii) - c). Sat $\Sigma 1=\{[y]|| y \mid \leq n\} \subseteq \Sigma \backslash\left(\Sigma_{2} \cup \Sigma_{3}\right)$. Sat $K 1=$ $\left\{[p, y] \mid(p, u, y) \in \delta_{1}(q, a, y),(q, a, y) \in K_{1} \times\left(\Sigma_{2} U\right.\right.$ $\left.\{\epsilon\}) \times G_{M_{1}}\right\} \subseteq K \backslash K_{I}$.

Forel $\not b i g$ er der blevet knyttet et nyt symbol [y] til hver mulig output streng, og vi har skabt en mangde af tilstande $[p, y]$, som husker hvilket output, der skulle udsendes samtidig med overgang til tilstanden.

$V i$ definerer nu fólgende acceptor

$D_{1}=\left(K_{1} \cup K^{\prime}, \Sigma_{2} \cup \Sigma_{1}, \delta_{2}, q_{0}, F\right)$ ved

$$
\begin{aligned}
& \text { i) } \forall(q, a, \gamma) \in K_{1} \times\left(\Sigma_{I} \cup\{\in\}\right) \times G_{M_{1}}: \\
& \mathbb{N}(p, u, y) \in \delta_{1}(q, a, \gamma) \\
& \text { ii) } \forall([p, y], u) \in \delta_{2}(q, a, y) \\
& \left(p, 1 \gamma^{\prime}\right)=\delta_{2}\left([p, y],[y], \gamma^{\prime}\right)
\end{aligned}
$$

Denne konstruktion tvinger os til at bruge reglerne fra i) og ii) alternerende. Yderligere er vi nodt til at starte med en regel af type i) og slutte med en af type ii). Heraf f $\phi \mid g e r$, at hvis $w \in L\left(D_{1}\right)$ kan $w$ skrives på formen

$$
w=a_{1}\left[y_{1}\right] a_{2}\left[y_{2}\right] \ldots a_{k}\left[y_{k}\right]
$$

hvor $a_{i} \in \Sigma_{1} U\{\varepsilon\}$ og $\left[y_{i}\right] \in \Sigma$.

Af konstruktionen folger det imidlertid også, at så er ( $a_{1} \ldots a_{k}$, $\left.y_{1} \ldots y_{k}\right) \in \operatorname{Gr}\left(M_{1}\right)$. Antag nemlig at $v i D_{1}$ anvender en regel 
fra i) på $a_{i}$. Den eneste måde hvorpå ii) kan anvendes er at vi vi moder [y], hvor y er output fra $M_{1}$ under anvendelse af den den tilsvarende regel her.

Lad omvendt $(x, y) \in G r\left(M_{1}\right)$. Det betyder pr: definition, at der findes en $f \phi l g e$ af konfigurationer for $M_{1} \quad k_{1} \ldots k_{\text {n }}$ med $k_{1}=\left(q_{0}, x, \varepsilon, \epsilon\right) k_{m}=(p, \varepsilon, \varepsilon, y) p \in F$, således at

$$
k_{i} \quad-k_{i+1} \quad 1 \leq i \leq m-1
$$

og $k_{i}, k_{i+1}$ er givet ved $k_{i}=\left(q^{\prime}, a_{i} w, \gamma, u\right) k_{i+1}=$ ( $p^{\prime}, w, \gamma^{\prime}, u v_{i}$ ) hvor $a_{i} \in\left(\Sigma_{2} \cup\{e\}\right) u, v_{i} \in \Sigma_{3}{ }^{*}$. Såkan vi skrive $x$ hhv. $y$ på formen $x=a_{1} \ldots a_{m} \quad y=v_{1} \ldots v_{m}$ men det betyder at $a_{1}\left[v_{1}\right] \ldots a_{1}\left[v_{m}\right] \in L\left(D_{1}\right)$ if $\phi$ lge konstruktionen af $D_{1}$.

Hvis $H$ er mængden af elementer $(a, y)$ for hvilke der findes $p, q, \gamma, u$, således at $\delta(p, a, \gamma) \ni(q, u, y)$, har vi vist at

$$
\begin{aligned}
L\left(D_{1}\right)=\left\{a_{1}\left[y_{1}\right] \ldots a_{k}\left[y_{k}\right] \mid\right. & \left(a_{1} \ldots a_{k}, y_{1} \ldots y_{k}\right) \in\left(T\left(M_{1}\right) \wedge\right. \\
& \left.\left(a_{i}, y_{i}\right) \in H, \quad 1 \leq i \leq k\right\}
\end{aligned}
$$

Da nu $M$ og $D$ var associerede, gælder at $D_{1} \in \mathcal{D}$ dvs. $L\left(D_{1}\right) \in \mathscr{L}(D)$.

Sæt $\Sigma_{1}=\left(\Sigma \cup \Sigma_{2}\right)$ og definer homomorfierne $h_{1}, h_{2}$ ved

$$
\begin{aligned}
& h_{1}(c)=\left\{\begin{array}{l}
c \text { hvis } c \in \Sigma_{2} \\
\epsilon \text { ellers }
\end{array}\right. \\
& h_{2}(c)=\left\{\begin{array}{l}
y \text { hvis } c=[y] \in \Sigma \\
\epsilon \text { ellers }
\end{array}\right.
\end{aligned}
$$

hvorefter vi har at

$$
\operatorname{Gr}(M)=\left\{\left(h_{1}(w), h_{2}(w)\right) \mid w \in L\left(D_{1}\right)\right\}
$$

b) Lad nu $L \in \mathscr{L}(\mathcal{D}) \quad L \subseteq \Sigma_{1}^{*}$ og lad $h_{1}: \Sigma_{1} * \rightarrow \Sigma_{2} *, h_{2}: \Sigma_{1} * \rightarrow \Sigma_{3}^{*}$ være to homomorfier. Definer $\Sigma$ l ved 
$\left.\Sigma I=\left\{\left[h_{1}(a), h_{2}(a)\right] \mid a \in \Sigma_{I}\right\} \subseteq \Sigma \backslash\left(\Sigma_{I} \cup \Sigma_{2} U_{3}\right)\right\} \quad$ og definer homomorfien $h^{\prime}: \Sigma_{1}^{*} \rightarrow \Sigma^{*}$ ved $h^{\prime}(a)=\left[h_{1}(a), h_{2}(a)\right]$. Da. $\mathscr{L}(\mathcal{D})$ er en AFL, gælder der at $h^{\prime}(L) \in \mathscr{L}(\mathcal{D})$.

Lad $D=\left\{K_{I}, \Sigma l, \delta_{1}, q_{0}, F\right\}$ vare en acceptor med $L(D)=h^{\prime}(L)$. Lad os definere en oversatter $M=\left\{K_{1}, \Sigma, \Sigma_{3}, \delta_{2}, q_{0}, F\right\}$ ved:

i) $\forall(q,[x, y], \gamma) \in K_{I} \times \Sigma l \times G_{D}$ :

$$
\mathbb{N}_{(p, u, y) \in \delta_{2}(q,[x, y], \gamma)}^{(p, u) \in \delta_{2}(q,[x, y], \gamma)}
$$

ii) $\prod_{(p, u, \epsilon) \in \delta_{2}(q, \epsilon, \gamma)}^{(p, u) \in \delta_{1}(q, \epsilon, \gamma)}$

Da $D$ og $M$ er associerede. gælder der at $M \in M, o g \operatorname{det} f \phi \mid-$ ger klart af konstruktionen at

$$
\operatorname{Gr}(M)=\left\{\left(\left[h_{1}\left(a_{1}\right), h_{2}\left(a_{1}\right)\right] \ldots\left[h_{1}\left(a_{n}\right), h_{2}\left(a_{n}\right)\right], h_{2}\left(a_{1} \ldots a_{n}\right)\right) \mid\right.
$$

Betragt f $\phi$ lgende homomorfi $h: \sum t * \sum_{2}^{*}$ defineret ved $h\left[h_{1}(a), h_{2}(a)\right]=h_{1}(a)$. Såfremt vi kan vise, at der findes $M_{1} \in \mathcal{M}$ således at

$$
\operatorname{Gr}\left(M_{1}\right)=\{(h(x), y) \mid(x, y) \in \operatorname{Gr}(M)\}
$$

er beviset for setning 3.6 fuldf $\phi r t$. Dette vises af det $f \phi \mid g e n d e$ Iemma.

Lemma 3.7 Lad $M$ være en AFTR og lad $M \in M$ vare en oversætter med $\operatorname{Gr}(M) \subseteq \Sigma_{1} * \times \Sigma_{2}^{*}$. Lad $h: \Sigma_{1} * \rightarrow \Sigma_{3^{*}}$ være en homomorfi. Der :findes da $M_{I} \in M$ således at

$$
\operatorname{Gr}\left(M_{1}\right)=\{(h(x), y) \mid(x, y) \in G r(M)\}
$$

Bevis Lad $M=\left\{K_{1}, \Sigma_{1}, \Sigma_{2}, \delta_{1}, q_{0}, F\right\}$ og sæt $\Sigma_{1}^{\prime}=\left\{a \in \Sigma_{1} \mid h(a)=e\right\}$. 
$\operatorname{Lad} \Sigma_{1} \backslash \Sigma_{1}^{\prime}=\left\{a_{1} \ldots a_{n}\right\}$ og $\operatorname{lad} h\left(a_{i}\right)=b_{i_{1}} \ldots b_{i_{k_{i}}} \quad 1 \leq i \leq n$. Lad endelig $K^{\prime}=\left\{p_{i_{I}}, \ldots, p_{i_{k_{i}}}\right\}_{i=1}^{n}$ være elementer $i K \backslash K_{1}$. Definer oversætteren $M_{1}=\left\{K_{1} \cup K_{1} \times K_{1}, \Sigma_{3}, \Sigma_{2}, \delta_{1}, q_{0}, F\right\}$ ved

i) $\prod_{\delta_{1}\left(q, \varepsilon, \gamma(q, \varepsilon, \gamma) \ni\left(q^{\prime}, u, y\right)\right.}^{\delta\left(q^{\prime}, u, y\right)}$

ii) $\prod_{\delta_{1}(q, \varepsilon, y) \ni\left(q^{\prime}, u, y\right)}^{\delta(q, y) \ni\left(q^{\prime}, u, y\right) \text { for } a \in \Sigma_{1}^{\prime}}$

iii) $\delta\left(q, a_{i}, y\right) \ni\left(q^{\prime}, u, y\right)$ for $a_{i} \in \Sigma_{1} \backslash \Sigma_{1}^{\prime}$

$$
\left\{\begin{array}{l}
\delta_{1}\left(q, b_{i_{1}}, \gamma\right) \ni\left(\left[q^{\prime}, p_{i_{1}}\right], 1 \gamma, \varepsilon\right) \\
\delta_{1}\left(\left[q^{\prime}, p_{i_{m}}\right], b_{i_{m}+1}, \gamma\right) \ni\left(\left[q^{\prime}, p_{i_{m}+1}\right], 1 \gamma, \varepsilon\right) \quad 1 \leq m \leq k_{i}-1 \\
\delta_{1}\left(\left[q^{\prime}, p_{i_{k}}\right], \varepsilon, \gamma\right) \ni\left(q^{\prime}, u, y\right)
\end{array}\right.
$$

Det er let at indse, at $M_{1}$ har de $\phi$ nskede egenskaber.

Bemærk, at det f $\phi r s t$ er $i$ beviset for lemma 3.7, at vi anvender at $\mathscr{L}(D)$ er en full AFL. Dette skal forstås på f $\phi$ lgende måde:

Da $\mathcal{M}$ og $\Phi$ er associerede, er Dom $(M) \in \mathscr{L}(\mathcal{D}) . M_{1}$ som konstrueret i lemma 3.7 har $\operatorname{Dom}\left(M_{1}\right)=h(\operatorname{Dom}(M))$, og da vi kræver, at $M_{I} \in M$ er det $n \phi d v e n d i g t$ at $h(\operatorname{Dom}(M)) \in \mathscr{L}(\mathcal{D})$ dvs. at $\mathscr{L}(\mathcal{D})$ er lukket over for vilkårlig homomorfi.

Hvis man fors $\phi g e r$ at definere familier af automater horende til en AFL som ikke er full, viser det sig (Ginsburg og Greibach [ 1969]), at disse stort set er karakteriseret ved at de kun må foretage et begranset antal sammenhangende $\epsilon$-trak. Af regel ii) i beviset for lemma 3.7 ses det, at selvom $M$ opfylder dette kriterium, er det ikke sikkert, at $M_{I} g \phi r$ det. Det er lige præcis det, der er problemet, når vi i afsnit 4 skal til at se på oversattelser defineret af line art begransede automater.

Definition 3.8 En push-down translator (PDTR) er et system $M=$ $\left\{K, \Sigma, \Gamma, \Delta, \delta, q_{0}, z_{0}, F\right\}$ hvor 
$K$ er en endelig mangde af tilstande

$\Sigma$ - - - $\quad$ - $\quad$ - inputsymboler

I - - - $\quad$ - staksymboler

$\Delta \quad-\quad$ - $\quad$ - outputsymboler

$\mathrm{q}_{\mathrm{O}} \in$ er startilistanden

$z_{0} \in I$ er stakbunden

$F \subseteq K$ er mængden af sluttilstande.

$\delta$ er en afbildning

$\delta: K \times(\Sigma \cup\{e\}) \times \Gamma \rightarrow \mathcal{P}_{\text {fin }}\left(K \times \Gamma^{*} \times \Delta^{*}\right)$

L er folgende relation på $K \times \Sigma^{*} \times \Gamma^{*} \times \Delta *$

$$
\begin{aligned}
& \text { for } a \in \Sigma\{€\} \text { er }(q, a x, \gamma Z, u) \mid-\left(p, x, \gamma \gamma^{\prime}, u v\right) \\
& \text { såfremt }\left(p, \gamma^{\prime}, v\right) \in \delta(q, a, Z) .
\end{aligned}
$$

Hvis $H^{*}$ er den reflexive; transitive lukning af - , definerer vi grafen af $M$ ved

$\operatorname{Gr}(M)=\left\{(x, y) \mid \exists p \in F:\left(q_{0}, x, z_{0}, \epsilon\right) H^{*}(p, \epsilon, \epsilon, y)\right\}$

Definition 3.9 En generaliseret sekventiel maskine (GSM) er et system $M=\left(K, \Sigma, \Delta, \delta, q_{0}, F\right)$ hvor $K, \Sigma, q_{0}, F$ er som $i$ def. 3.8. $\delta$ er en afbildning

$$
\delta: K \times(\Sigma \cup\{\epsilon\}) \rightarrow P_{\text {fin }}(K \times \Delta *)
$$

- er folgende relation på $K \times \sum^{*} \times \Delta *$

$$
\begin{aligned}
& \text { for } a \in \Sigma \cup\{\epsilon\} \quad(q, a x, u) \mid-(p, x, u v) \\
& \text { såfremt }(p, v) \in \delta(q, a)
\end{aligned}
$$

- ${ }^{*}$ er igen den reflexive transitive lukning af $/$ og vi sætter grafen for $M$ til

$$
\operatorname{Gr}(M)=\left\{(x, y)\left|\exists p \in F:\left(q_{0}, x, \epsilon\right)\right| *(p, \varepsilon, y)\right\}
$$


Ginsburg og Greibach [1969] viser, hvorledes familierne af pushdown automater og endelige automater hver for sig danner en AFA. Ved hjælp heraf er det trivielt at vise at familierne af PDTR og GSMler danner AFTR'er, men det giver straks f $\phi$ Ignede corollar til sætning 3.6.

Corollar 3.10 Lad $\Sigma, \Delta$ være to alfabeter og lad $T \subseteq \Sigma * x \Delta *$. der findes da en PDTR (GSM) $M$ således, at $G r(M)=T$ hvis og kun hvis der findes et sprog $L \in C F$ (REG) og to homomorfier $h_{1}, h_{2}$ således at

$$
T=\left\{\left(h_{I}(w), h_{2}(w)\right) \mid w \in L\right\}
$$

Lad os vende os mod en anden måde at karakterisere grafer for PDTR og GSMler, nemlig v. hj. a. grammatikker. Aho og Ullman [1969a] indf $\phi$ rer begrebet syntaxdirigeret oversættelses skema (SDTS). Her er vi kun interesseret i den simpleste udgave af SDTS, nemlig det såkaldte simpel syntax dirigeret over sættelses skema (SSDTS).

Definition 3.11 En SSDTS er et system $G=(V, \Sigma, \Delta, R, S)$, hvor $V$ er en endelig mangde af hjælpesymboler, $\Sigma, \Delta$, er endelige mangder af hhv. input-og outputsymboler, $S \in V$ er startsymbolet og $R$ er en endelig mangde af regler. Vi har som sædvanlig $\vee \cap(\Sigma \cup \Delta)=\phi$.

En form er et par $(\alpha, \beta)$ med $\alpha \in(V \cup \Sigma)^{*}, \beta \in(V \cup \Delta)^{*}$ som opfylder at hvis vi betragter homomorfien h: $(V \cup \Sigma \cup \Delta)^{*} \rightarrow V^{*}$ defineret ved

$$
h(A)=\left\{\begin{array}{l}
A \text { hvis } A \in V \\
\epsilon \text { ellers }
\end{array}\right.
$$

så er $h(\alpha)=h(\beta)$.

En regel er et element af formen $A \rightarrow(\alpha, \beta)$ hvor $A \in V$ og $(\alpha, \beta)$ er en form. 
$(\alpha, \beta)$ er altså en form hvis og kun hvis $\alpha$ og $\beta$ indeholder de samme hjælpesymboler i samme rækkef $\phi$ lge.

Definition 3.12 Lad $G$ være en SSDTS. Lad os definere relationen $\Rightarrow$ mellem former for $G$. For former $\left(\alpha_{1}, \beta_{1}\right)\left(\alpha_{2}, \beta_{2}\right)$ skriver vi

$$
\left(\alpha_{1}, \beta_{2}\right) \Rightarrow\left(\alpha_{2}, \beta_{2}\right)
$$

såfremt vi kan skrive $\left(\alpha_{1}, \beta_{1}\right)$ på formen ( $\left.\omega_{1} A \delta_{1}, \omega_{2} A \delta_{2}\right)$ med $h\left(w_{1}\right)=h\left(\omega_{2}\right), h\left(\delta_{1}\right)=h\left(\delta_{2}\right)$ og vi kan skrive $\left(\alpha_{2}, \beta_{2}\right)$ på formen $\left(\begin{array}{llllll}\omega_{1} & \gamma_{1} & \delta_{1} & \omega_{2} & \gamma_{2} & \delta_{2}\end{array}\right)$ og der gæider at $A \rightarrow\left(\gamma_{1}, \gamma_{2}\right)$ er en regel. Hvis $\stackrel{*}{\Rightarrow}$ er den reflexive transitive lukning af $\Rightarrow$ vil vi ved den simple syntax dirigerede oversattelse (SSDT) defineret af $G$ forstå mængden

$$
T(G)=\{(x, y) \mid(s, s) \stackrel{*}{\Rightarrow}(x, y)\}
$$

Når vi anvender en regel i en SSDTS erstatter vi altså samtidigt samme forekomst af samme hjælpesymbol $i$ en form med regelens hфjreside.

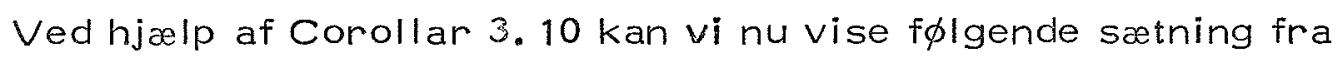
Aho og Ullman [1969a].

Sætning 3.13 Lad $\Sigma, \Delta$ være alfabeter og lad $T \subseteq \Sigma * x \Delta *$. Der findes da en PDTR $M$ således at $T=G r(M)$ hvis og kun hvis der findes en SSDTS $G=(V, \Sigma, \Delta, R, S)$ således at $T=T(G)$.

Bevis Lad $M$ vare en PDTR med $G r(M)=T$. Lad $L \in C F, h_{I}, h_{2}$ homomorfier så

$$
\operatorname{Gr}(M)=\left\{\left(h_{1}(w), h_{2}(w)\right) \mid w \in L\right\}
$$

og lad $G_{L}=\left(V_{N}, V_{T}, P, S\right)$ være en grammatik for $L$. Konstruer

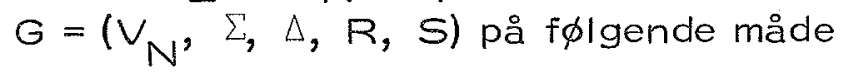


Hvis $A \rightarrow x_{1} B_{1} \ldots x_{n} B_{n} x_{n+1} \quad n \geq 0$

er et element i $P$ er

$A \rightarrow\left(h_{1}\left(x_{1}\right) B_{1} \ldots h_{1}\left(x_{n}\right) B_{n} h_{1}\left(x_{n+1}\right), h_{2}\left(x_{1}\right) B_{1} \ldots h_{2}\left(x_{n}\right) B_{n} h_{2}\left(x_{n+1}\right)\right)$

element i R.

Det er klart at

$$
T=\left\{\left(h_{1}(w), h_{2}(w)\right) \mid w \in L\right\}=T(G)
$$

Lad omvendt $G=(V, \Sigma, \Delta, R, S)$ være en SSDTS. Konstruer en grammatik $G_{L}=\left(V, V_{T}, P, S\right)$ på f $\phi \mid$ gende måde

hvis $A \rightarrow\left(x_{1}^{i} B_{1}^{i} \ldots x_{n_{1}}^{i} B_{n_{i}}^{i} x_{n_{i}+1}^{i}, y_{1}^{i} B_{1}^{i} \ldots y_{n_{i}}^{i} B_{n_{i}}^{i} y_{n_{i}+1}^{i}\right)$

er den ilte regel $i R$ er

$$
A \rightarrow C_{1}^{i} B_{1}^{i} \ldots C_{n_{i}}^{i} B_{n_{i}}^{i} C_{n_{i}+1}^{i}
$$

en produktion i P. $\left\{c_{1}^{i} \ldots c_{n+1}^{i}\right\}$ er forskellige symboler for hver regel $i$ R. Sæt $C=U\left\{c_{1}^{i}, \ldots c_{\pi_{1}}^{i}{ }_{i}\right\}$ og definer to homomorfier $h_{1}: C^{*} \rightarrow \sum^{*}, h_{2}: C^{*} \rightarrow \Delta *$ ved

$$
h_{1}\left(c_{j}^{i}\right)=x_{j}^{i} \quad h_{z}\left(c_{j}^{i}\right)=y_{j}^{i}
$$

Lad $M$ være den PDTR, som har

$$
G r(M)=\left\{\left(h_{1}(w), h_{2}(w)\right) \mid w \in L\left(G_{L}\right)\right\}
$$

Det er klart at $\operatorname{Gr}(M)=T(G)$.

HVis vi definerer en analogi til SSDTS for regulære grammatikker ved at en form har udseendet

$$
(\alpha, \beta)=(x A, y A) \quad x \in \Sigma^{*}, y \in \Delta^{*}, A \in V U\{\epsilon\}
$$


og på indlysende vis definerer analogien til SSDT, er det lige ud ad landevejen at vise en sætning for GSMier, der er analog til sætning 3.13 .

Hermed sluttes omtalen af oversættelsesbegrebet på grundlag af context-free sprog. Aho og Ullman [1969] omhandler almindelig syntax dirigeret oversættelse dvs. oversættelse, hvor komponenterne $i$ en form $(\alpha, \beta)$ stadig skal indeholde de samme hjælpesymboler, men hvor rækkef $\phi \mid g e n ~ i ~ k$ kan være en permutation af rækkefolgen i $\alpha$. Aho og Ullman [1971] generaliserer begrebet SDTS ved til hver knude $i$ et derivationstræ for et ord at knytte ikke én men flere semantiske variable. 


\section{AFSNIT 4}

LINEAET BEGRANSEDE AUTOMATER OG LINEAERT BEGRAN= SEDE OVERSAETTERE.

Der findes rundt om i litteraturen forskellige definitioner af lineær $t$ begrænsede automater. Disse er dog alle ækvivalente $i$ den forstand, at Klassen af sprog, der accepteres, er CS. Her vil vi bruge næsten samme definition som i Ginsburg og Rose [ 1966].

Definition 4.1 En lineært begrænset automat (LBA) er et system $M=\left(K, \Sigma, T, \delta, q_{0}, F\right)$ hvor

$K$ er en endelig ikke tom mængde af tilstande

$\Sigma$ - $\quad$ - $\quad$ - $\quad$ - $\quad$ - _ _ - inputsymboler

$I \supseteq \sum$ er en endelig ikke tom mængde af tapesymboler

$q_{0} \in K$ er starttilstanden

$F \subseteq K$ er mangden af sluttilstande

$\delta$ er en afbildning

$$
\delta: K \times I \rightarrow \mathcal{P}_{\text {fin }}(K \times \Gamma \times\{L, N, R\})
$$

Definition 4.2 Lad $M=\left(K, \Sigma, \Gamma, \delta, q_{0}, F\right)$ være en LBA. I- er en relation på $\Gamma^{*} K \Gamma^{*}$ defineret ved

$$
\begin{aligned}
& \text { for } u, v, \in \Gamma^{*}, c \in \Gamma \text { er } \\
& \begin{array}{l}
\text { i) ucpav I- uqcbv } \\
\mathbb{I}_{\delta(p, a) \ni(q, b, L)}
\end{array} \\
& \begin{array}{l}
\text { ii) upav } 1-\text { uqbv } \\
\mathbb{I}_{\delta(p, a) \ni(q, b, N)}
\end{array} \\
& \text { iii) upav } 1-\text { ubqv } \\
& \mathbb{v}_{\delta}(p, a) \ni(a, b, R)
\end{aligned}
$$


Hvis $H^{*}$ er den reflexive, transitive lukning af - vil vi ved sproget accepteret af $M$ forstå mangden

$$
T(M)=\left\{w \in \Sigma^{+} \mid B q \in F, \alpha \in \Gamma^{*}: q_{0} w H^{*} \alpha q\right\}
$$

Bemærk, at der for alle LBA $M$ gælder, at $\varepsilon \notin T(M)$. (jfr. side 1.2)

Definition 4.3 En lineært begræenset oversætter (LBTR) er et system $M=\left(K, \Sigma, T, \Delta, \delta, q_{0}, F\right)$ hvor $K, \Sigma, T, q_{0}, F$ er som $i$ def. $4.1 \mathrm{og}$ der yderligere galder

$\Delta$ er en endelig ikke tom mæengde af output symboler

$\delta$ er en afbildning

$$
\delta: K \times \Gamma \rightarrow P_{\text {fin }}\left(K \times \Gamma \times(L, N, R) \times \Delta^{*}\right)
$$

Definition 4.4 Lad $M=\left(K, \Sigma, \Gamma, \Delta, \delta, q_{0}, F\right)$ være en LBTR. - er en relation på $\Gamma^{*} K \Gamma^{*} \times \Delta^{*}$ defineret ved

for $u, v \in \Gamma^{*}, c \in \Gamma, x, y \in \Delta^{*}$ er

i) $\prod_{\delta(p, a) \ni(q, b, L, y)}^{(\text {ucpav, } x) \mid-(\text { uqcbv, } x y)}$

ii) $\begin{array}{r}\text { (upav, } x) \mid-(u q b v, x y) \\ \delta(p, a) \ni(q, b, N, y)\end{array}$

iii) $\Uparrow_{\delta(p, a)}^{(u p a v, x)-(u b q v, x y)}$

Hvis $L^{*}$ igen er den reflexive, transitive lukning af $H_{-}$vil vi ved grafen for $M$ forstå mængden

$$
\operatorname{Gr}(M)=\left\{(w, y) \in \Sigma^{+} \times \Delta^{*} \mid \exists q \in F, \beta \beta \in \Gamma^{*}:(q, w, \epsilon) W^{*}(\beta q, y)\right\}
$$

Bemærkning 4.5 Af ovenstående definitioner folger det, at hvis $M$ er en LBTR, er Dom $(M) \in C S$. 
Som kommentar til def. 4. 1-4.4 kan det siges, at der ingen steder optrader de "begransnings marker", som er velkendte fra andre definitioner af LBAier. Sådanne steder optræder der to specielle symboler, f.eks. $\$$ og $\$$ som bruges til at afgrænse den del af båndet, hvorpå LBAien må arbejde. Havde vi taget begrænsningsmarker med $i$ vor definition af en LBA $M$, skulle vi have lagt $f \phi i-$ gende krav på $\delta$

i)

$$
\begin{aligned}
& \Downarrow_{b=\phi}^{\delta(q, \phi) \ni(p, b, x)} \\
& b=\varnothing x=R
\end{aligned}
$$

ii)

$$
\begin{aligned}
& \| \delta(q, \$) \ni(p, b, x) \\
& b=\$ \wedge x=L
\end{aligned}
$$

og vi ville $i$ så fald have defineret $T(M)$ ved

$$
T(M)=\left\{w \in \Sigma^{+} \mid \beta q \in F, B \beta \in \Gamma^{*}: \phi q_{0} w \$ H^{*} \neq \beta q \$\right\}
$$

Ginsburg og Rose [1966] har imidlertid vist, at man ikke opnår noget ekstra ved at medtage begrænsningsmærker, dvs. den klasse af sprog der accepteres er i begge tilfalde klassen af context sensitive sprog. I det f $\phi$ lgende kan vi derfor $i \mathrm{fl}$ ang bruge den af de to definitioner af LBA og LBTR, der "passer" bedst til lejligheden.

I lys af ovenstående bemæerkninger kan vi nu give et simpelt bevis for et resultat (Ginsburg og Rose [1966]), der viser en af forskellene på grafer for LBTRler og de $i$ afsnit 3 omtalte oversættere.

Satning 4.6 Lad $L \in R E . L \subseteq \Sigma^{*}$. Der findes da en LBTR $M$, således at $\operatorname{Ran}(M)=L$.

Bevis Lad $L_{1}$ vare som i lemma 2.3 og lad $M_{1}=(K, \Sigma \cup\{\#\}, T$, $\left.\delta_{1}, q_{0}, F\right)$ være en LBA med begrænsningsmærker, således at $L_{1}=T\left(M_{1}\right)$. Definer en LBTR $M=\left(K \cup\left\{p_{0}, p_{1}, p_{2}\right\}, \Sigma \cup\{\#\}, \Gamma U I \times \Sigma\right.$, $\left.\sum, \delta, q_{0}, p_{2}\right)$ ved for $a, q^{\prime} \in R, a \in \Sigma, b, c \in \Gamma, x \in\{L, N, R\}$

i) $\delta\left(q_{0}, a\right)=\left(p_{0},(a, a), R, \dot{e}\right)$

ii) $\delta\left(p_{0}, a\right)=\left(p_{0},(a, a), R, e^{\prime}\right)$

iii) $\delta\left(p_{0}, \$\right)=\left(p_{0}, \$, L, i\right)$

iv) $\delta\left(p_{\circ},(a, a)\right)=\left(p_{\circ},(a, a), L, \varepsilon\right)$ 


$$
\begin{aligned}
& \text { v) } \delta\left(p_{0}, \phi\right)=\left(q_{0}, \phi, R, e\right) \\
& \text { vi) } \delta(q,(a, b)) \ni\left(q^{i},(c, b), x, €\right) \\
& \mathbb{I}_{\delta_{1}}(q, b) \ni\left(q^{i}, c, x\right) \\
& \text { vii) } \delta(q, \$)=\left(p_{1}, \$, L, e\right) \text { for } q \text { e } F \\
& \text { viii) } \delta\left(p_{1},(a, b)\right)=\left(p_{1},(a, b), L, e\right) \\
& \text { ix) } \delta\left(p_{1}, \phi\right)=\left(p_{2}, \phi, R, €\right) \\
& \text { x) } \delta\left(p_{2},(a, b)\right)=\left(p_{2}, a, R, b\right) \quad b \in \Sigma \\
& \quad \delta\left(p_{2},(a, \#)\right)=\left(p_{2}, a, R, e\right)
\end{aligned}
$$

$M$ starter med at gemme en kopi af input $i$ andenkomponenten af sym-

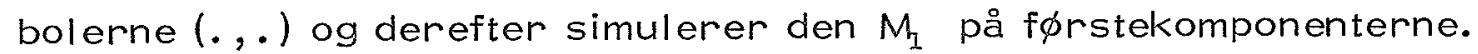
Safremt $M_{1}$ accepterer kopierer $M_{1}$ alle oprindelige inputsymboler på nær \#. Det er klart at $\operatorname{Ran}(M)=L$.

I det fфigende vil vi se på, i hvor hфj grad resultaterne fra afsnit 3 kan overf $\phi r e s$ til LBTRler. Inden vi er helt klar til det, skal vi imidlertid have endnu en bemærkning om begrænsningsmærker.

Definition 4.7 En context sensitiv grammatik med begræensningsmarker, er et system $G=\left(V_{N}, V_{T}, P, S, \#\right)$ hvor

i) $\quad \# \notin V_{T}$

ii) $\left(V_{N}, V_{T} \cup\{\#\}, P, S\right)$ er en context sensitiv grammatik

iii) For enhver produktion $\alpha \rightarrow \beta$ galder der at $\alpha, \beta$ samtidigt er på en af folgende former $\$ \delta, \delta, \delta$ hvor $\delta \in V^{*}$

Ved sproget genereret af $G$ vil vi forstå mængden

$$
\# L=L(G)=\{\# w \# \mid \text { 草 }
$$

Landweber [1963] har vist, at $L$ er context sensitiv hvis og kun hvis \# $L$ genereres af en context sensitiv grammatik med begrænsningsmærker, således at vi også her kan anvende den form, der passer bedst $i$ det givne tilfælde.

Vi kan nu vise f $\phi$ lgende: 
Satning 4.8 Lad $M=\left(K, \Sigma, \Gamma, \Delta, \delta, q_{0}, F\right)$ vare en LBTR og lad $\Lambda$ være et symbol med $\Lambda \notin(\Sigma U \Delta)$. Der findes et context sensitivt sprog $L \subseteq(\Sigma U \Delta U\{\Lambda\})^{*}$ og to homomorfier $h_{\perp}$ : $(\Sigma \cup \Delta \cup \Lambda)^{*} \rightarrow \Sigma^{*}, h_{2}:(\Sigma \cup \Delta \cup \Lambda)^{*} \rightarrow \Delta^{*}$ således at

$$
G r(M)=\left\{\left(h_{I}(w), h_{2}(w)\right) \mid w \in L\right\}
$$

Bevisetfor sætning 4.8 er temmelig teknisk, så vi viser det v.hj.a. et par lemmaer.

Lemma 4.9 Lad $M=\left(K, \Sigma, \Gamma, \delta, q_{O}, F\right)$ være en LBA. Konstruer en context sensitiv grammatik $G=(\Gamma \times \Sigma \cup K \times \Gamma \times \Sigma, \Sigma, P, S, \sharp)$ hvor $P$ er folgende produktioner

$$
\begin{aligned}
& \text { i) } S \rightarrow S(a, a) \text { for alle } a \in \Sigma \\
& S \rightarrow(a, a) \\
& \text { ii) } \quad \#(a, a) \rightarrow \#\left[q_{0}, a, a\right] \\
& \text { iii) } \hat{(}(p, b, L) \in \delta(q, a) \\
& \Downarrow(d, e)[q, a, c] \rightarrow[p, d, e](b, c) \\
& \text { iv) } \Uparrow(p, b, N) \in \delta(q, a) \\
& \sqrt{ }[\mathrm{q}, \mathrm{a}, \mathrm{c}] \rightarrow[\mathrm{p}, \mathrm{b}, \mathrm{c}] \\
& \text { v) } \mathbb{\Downarrow}_{[a, b, R) \in \delta(q, a) \quad \text { for } p \in F}^{(p, c](d, e) \rightarrow(b, c)[p, d, e]} \\
& \text { vi) } \begin{array}{l}
\Downarrow^{(p, b, R) \in \delta(q, a)} \quad \text { for } p \in F \\
{[q, a, c] \# \rightarrow c \text { 柆 }}
\end{array} \\
& \text { vii) }(d, e) c \rightarrow e c
\end{aligned}
$$

og hvor, a,b, $d \in \Gamma, c, e \in \Sigma, p, q \in K$.

Hvis $L(G)=\# L$ galder der, at $L=T(M)$.

Bevis. Ved hjalp af de context free produktioner i) genererer grammatikken en streng af formen $\#\left(a_{1}, a_{1}\right) \ldots\left(a_{n}, a_{n}\right) \#$ hvorefter vi tvinges til at bruge produktion $n r$. ii) til at få $\#\left[q_{0}, a_{1}, a_{1}\right] \ldots\left(a_{n}, a_{n}\right)$. 
Herefter simulerer grammatikken automatens bevægelser idet den opererer på f $\phi$ rstekomponenten i hjælpesymbolerne $(\cdot, \cdot)$. Anden komponenten bruges til at huske, hvad der oprindeligt stod på denne plads. Såfremt automaten accepterer strengen $a_{1} \ldots a_{n}$, dvs. $\exists q \in F$ og $\beta \beta \in \Gamma^{*}:\left(q_{0} a_{1} \ldots a_{n}\right) H^{*}(\beta q)$ sikrer produktionerne $v i)$ og $v i i)$, at vi slutter med \# $a_{1} \ldots a_{n} \#$. Vi vil ikke give et mere formelt bevis, idet det skulle være klart, at konstruktionen virker.

Definition 4.10 En LBTR $M=\left(K, \Sigma, T, \Delta, \delta, q_{0}, F\right)$ siges at vare $\epsilon$-fri såfremt

$$
\|_{y \neq \varepsilon}^{(p, b, x, y) \in \delta(q, a)}
$$

Lemma 4.11 Lad $M=\left(K, \Sigma, \Gamma, \Delta, \delta, q_{0}, F\right)$ være en $\varepsilon$-fri LBTR. Så er Ran (M) context sensitiv.

Bevis Da $M$ er $\varepsilon-f r i$, kan vi antage, at $y \quad i(p, b, x, y) \in \delta(q, a)$ tilhorer $\Delta$, idet vi altid v.hj.a. en $\varepsilon$-fri homomorfi, som bevarer context sensitive sprog, kan transformere tilbage til det oprindelige. $\checkmark i$ vil konstruere en context sensitiv grammatik for Ran (M). Lad $\bar{\Delta}=\{\bar{A} \mid A \in \Delta\}$ og sæt $G=(\Gamma \cup\{\Gamma \times \Delta\} \cup\{K \times \Gamma\} \cup \bar{\Delta}, \Delta, P, S, \#)$ hvor $P$ er folgende produktioner

$$
\begin{aligned}
& \text { i) } S \rightarrow S a \text { for alle } a \in \Sigma \\
& S \rightarrow a \\
& \text { ii) } \quad \# a \rightarrow \#\left[a_{0}, a\right] \text { for alle } a \in \Sigma
\end{aligned}
$$

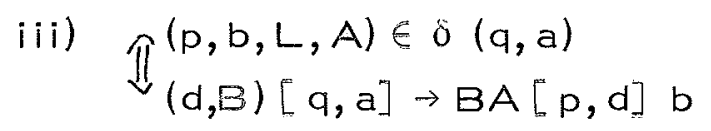

$$
\begin{aligned}
& \text { iv) } \mathfrak{d}_{[q, a] \rightarrow A[p, b]}^{(p, b, N, A) \in \delta(q, a)} \\
& \text { v) } \mathbb{1}_{[q, a] d \rightarrow(b, A)[p, d]}^{(p, b, R, A) \in \delta(q, a)} \\
& \text { vi) }(a, A) B \rightarrow A(a, B)
\end{aligned}
$$

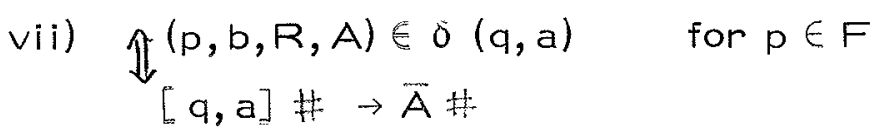


viii) $\quad(a, A) \bar{B} \rightarrow \bar{A} B$

$A \bar{B} \rightarrow \bar{A} B$

ix) \# $\quad \# \rightarrow \#$

hvor $a, b, d \in \Gamma$ og $A, B \in \Delta$

Med $L(G)=\# L$ er $L=\operatorname{Ran}(M)$. Bevis herfor findes $i$ Ginsburg og Rose [ 1966] , og vi vil ikke gå ind på det her. Argumentet er stort set det samme som i lemma 4.9.

En fornemmelse af, hvordan konstruktionen virker, fås af f $\phi$ lgende eksempel.

Eksempel 4.12 Næsten ethvert eksempel, der har noget med context sensitive sprog at gфre, har traditionelt også noget med sproget

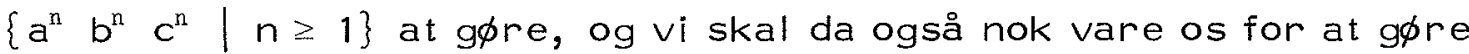
undtagelser her.

Betragt $f \phi \mid$ gende LBTR $M=\left(K, \Sigma, I, \Delta, \ddot{0}, q_{0}, F\right)$ hvor

$$
\begin{aligned}
& K=\left\{q_{0}, q_{1}, q_{2}, q_{3}, q_{4}\right\} \\
& \Sigma=\{a, b, c\} \\
& \Gamma=\{a, b, c, \bar{a}, \bar{b}, \bar{c}\} \\
& \Delta=\{1,2,3,4,5,6,7,8,9, A, B, C, D, E, F\} \\
& F=\left\{q_{4}\right\}
\end{aligned}
$$

og 0 er defineret ved

$$
\begin{aligned}
\text { I } & \delta\left(q_{0}, a\right) & =\left(q_{1}, \bar{a}, R, 1\right) \\
\text { II } & \delta\left(q_{1}, a\right) & =\left(q_{1}, \bar{a}, R, 2\right) \\
\text { III } & \delta\left(q_{1}, \bar{b}\right) & =\left(q_{2}, \bar{b}, R, 3\right) \\
\text { IV } & \delta\left(q_{1}, \bar{b}\right) & =\left(q_{1}, \bar{b}, R, 4\right) \\
\text { V } & \delta\left(q_{2}, b\right) & =\left(q_{2}, \bar{b}, R, 5\right) \\
\text { VI } & \delta\left(q_{2}, \bar{c}\right) & =\left(q_{2}, \bar{c}, R, 6\right)
\end{aligned}
$$




$$
\begin{array}{ll}
\text { VII } & \delta\left(q_{2}, c\right)=\left(q_{3}, \bar{c}, L, 7\right) \\
\text { VIII } & \delta\left(q_{3},\left\{\begin{array}{l}
\frac{b}{b} \\
a \\
c
\end{array}\right\}\right)=\left(q_{3}, L,\left\{\begin{array}{l}
\frac{b}{b} \\
\frac{a}{c}
\end{array}\right\},\left\{\begin{array}{l}
A \\
B \\
c \\
D
\end{array}\right\}\right) \\
\text { IX } & \delta\left(q_{3}, \bar{a}\right)=\left(q_{0}, \vec{a}, R, 8\right) \\
\times & \delta\left(q_{0}, \bar{b}\right)=\left(q_{4}, \bar{b}, R, g\right) \\
\times I & \delta\left(q_{4},\left\{\frac{\vec{b}}{\bar{c}}\right\}\right)=\left(q_{4},\left\{\frac{\bar{b}}{\bar{c}}\right\}, R,\left\{\begin{array}{l}
E \\
F
\end{array}\right)\right.
\end{array}
$$

Det er nemt at indse, at $\operatorname{Dom}(M)=\left\{a^{n} b^{n} c^{n} \mid n \geq 1\right\}$ og at $M$ virker i overensstemmelse med f $\phi$ lgende algoritme

1: $\quad$ if symbol $=$ a then $\downarrow \bar{a}$ else if symbol $=\bar{b}$ then goto $2 ; \rightarrow$;

while symbol $=a \vee \vec{b}$ do $\rightarrow$;

if symbol $=\mathrm{b}$ then $\downarrow \overline{\mathrm{b}} ; \rightarrow$;

while symbol $=\mathrm{b} \vee \overline{\mathrm{c}}$ do $\rightarrow$;

if symbol $=c$ then $\downarrow \vec{c}$; $\leftarrow$;

while symbol $=\mathrm{b} \vee \overrightarrow{\mathrm{b}} \vee \mathrm{a} \vee \overline{\mathrm{c}}$ do $\leftarrow$;

if symbol $=\overline{\mathrm{a}}$ then $\rightarrow$;

goto 1 ;

2: $\quad$ while symbol $=\bar{b} \vee \vec{c}$ do $\rightarrow$;

symbol betegner det symbol, $M$ i $\phi$ jeblikket læser; $\downarrow \times$ betyder, at $x$ skrives, $\rightarrow$, - betegner et ryk hhv. til hфjre og til venstre. Der er ikke taget hensyn til output.

Lemma 4. 11 giver folgende grammatik for Ran $(M)$ :

i) $S \rightarrow \mathrm{Sa}, \mathrm{s} \rightarrow \mathrm{Sb}, \mathrm{s} \rightarrow \mathrm{Sc}$

$S \rightarrow a, S \rightarrow b, S \rightarrow c$

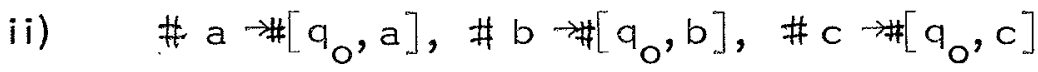

$\begin{array}{rlrl}\text { I } & {\left[q_{0}, a\right] d} & \rightarrow(\bar{a}, 1)\left[q_{1}, d\right] \\ \text { II } & {\left[q_{1}, a\right] d} & \rightarrow(a, 2)\left[q_{1}, d\right] \\ \text { III } & {\left[q_{1}, b\right] d} & \rightarrow(\bar{b}, 3)\left[q_{2}, d\right] \\ \text { IV } & {\left[q_{1}, \bar{b}\right] d \rightarrow(\bar{b}, 4)\left[q_{1}, d\right]} \\ \text { V } & {\left[q_{2}, b\right] d \rightarrow(b, 5)\left[q_{2}, d\right]} \\ \text { VI } & {\left[q_{2}, \bar{c}\right] d \rightarrow(\bar{c}, 6)\left[q_{2}, d\right]} \\ \text { VII } & (d, z)\left[q_{2}, c\right] \rightarrow z 7\left[q_{3}, d\right] \bar{c}\end{array}$




$$
\begin{aligned}
& \text { VIII }(d, z)\left[q_{3},\left\{\begin{array}{c}
\frac{b}{b} \\
a \\
c
\end{array}\right\}\right] \rightarrow z\left\{\begin{array}{l}
A \\
B \\
c \\
D
\end{array}\right\},\left[q_{3}, d\right]\left\{\begin{array}{c}
\frac{b}{b} \\
\frac{a}{c}
\end{array}\right\} \\
& \text { ix } \quad\left[q_{3}, \bar{a}\right] d \rightarrow(\bar{a}, 8)\left[q_{0}, d\right] \\
& \times \quad\left[q_{0}, \bar{b}\right] d \rightarrow(\bar{b}, 9)\left[q_{4}, d\right] \\
& \times I \quad\left[a_{4},\left\{\frac{\vec{b}}{\bar{c}}\right\}\right] d \rightarrow\left(\left\{\frac{\vec{b}}{\bar{c}}\right\},\left\{\frac{E}{F}\right\}\right)\left[a_{4}, d\right] \\
& \text { vi) } \quad(d, Z) Y \rightarrow Z(d, Y) \\
& \text { vii) }\left[a_{0}, \bar{b}\right] \# \rightarrow \bar{g} \#,\left[q_{4}, \bar{b}\right] \# \rightarrow \bar{E} \#,\left[a_{4}, \bar{c}\right] \# \rightarrow \bar{F} \# \\
& \text { viii) } \quad(d, Z) \bar{Y} \rightarrow \bar{Z} Y \\
& Z \bar{Y} \rightarrow \bar{Z} Y \\
& \text { ix) } \quad \# \bar{Z} \rightarrow \# Z
\end{aligned}
$$

hvor $d \in \Gamma \operatorname{og} Z, Y \in \Delta$

Lad os prфqve at betragte en derivation af output fra ordet abc ethvert st $\phi$ rre eksempel svulmer voldsomt op -.

$$
\begin{aligned}
& \text { (i) } * \text { \#\# } \\
& \text { (ii) \#abc 柿 } \\
& \text { i \#[q,a] bc \# } \\
& \text { 拐( } \bar{a}, 1)\left[q_{1}, b\right] c \\
& \text { III \#(a, 1) }(\bar{b}, 3)\left[a_{2}, c\right] \text { \# } \\
& \text { vil \#( } \\
& \text { VIII \#13( } \bar{a}, 7)\left[q_{3}, \bar{b}\right] \bar{c} \text { 林 } \\
& \begin{array}{ll}
\text { IX } & \# 1378\left[q_{3}, \bar{a}\right] \bar{b} \bar{c} \# \\
x & \# 137 B(\bar{a}, 8)\left[a_{0}, \bar{b}\right] \bar{c} \#
\end{array} \\
& \begin{array}{ll}
\text { (vii) } & \# 137 B(\bar{a}, 8)(\bar{b}, 9)\left[q_{4}, \bar{c}\right] \# \\
\text { (viii) } & \# 137 B(\bar{a}, 8)(\bar{b}, 9) \vec{F} \# \\
\text { (viii) } \# 137 B(\bar{a}, 8) \overrightarrow{9} F \#
\end{array} \\
& \text { (viii) \#137B } \overline{8} 9 \mathrm{~F} \# \\
& \text { (viii)* \#T37889F\# } \\
& \text { \#1378 } 89 F
\end{aligned}
$$


Hver streng $i$ eksemplet deriveres af den, der står ovenover under en eller flere $\left(^{*}\right)$ anvendelser af den eller de regler, hvis nummer står mellem strengene.

Det fremgår, at de to grammatikker i lemma 4.9 og lemma 4.11 "strukturelt" set er ens. Dette anvendes nu til at vise f $\phi$ lgende

Lemma 4.13 Lad $M=\left(K, \Sigma, \Gamma, \Delta, \delta, q_{0}, F\right)$ være en $\epsilon$-fri LBTR. Sproget

$$
D=\{w y \mid(w, y) \in G r(M)\}
$$

er da context sensitivt.

Bevis Konstruer følgende context sensitive grammatik $G=$ $(\{\Gamma \times \Sigma\} \cup\{\Gamma \times \Sigma \times \Delta\} \cup\{K \times \Gamma \times \Sigma\} \cup \vec{\Delta} \cup \hat{\Sigma}, \Sigma \cup \Delta, \mathbf{P}, \mathbf{S}, \#)$, hvor vi har sat $\vec{\Delta}=\{\vec{A} \mid A \in \Delta\}, \hat{\Sigma}=\{\hat{c} \mid c \in \Sigma\}$ og hvor $P$ er f $\phi \mid-$ gnede produktioner

$$
\begin{aligned}
& \text { i) } \quad s \rightarrow s(a, a) \\
& S \rightarrow(a, a) \quad \text { for alle } a \in \Sigma \\
& \text { ii) } \quad \#(a, a) \rightarrow \#\left[q_{0}, a, a\right] \text { for alle } a \in \Sigma \\
& \text { iii) } \prod_{(p, b, L, A) \in \delta(a, a)} \\
& \Downarrow(d, e, B)[q, a, c] \rightarrow B A[p, d, e](b, c) \\
& \text { iv) } \mathbb{\prod}_{[q, a, c] \rightarrow A[p, b, c]}^{(p, b, A) \in O(q, a)} \\
& \text { v) } \hat{(p, b, R, A) \in \delta(q, a)} \\
& \Downarrow[q, a, c](d, e) \rightarrow(b, c, A)[p, d, e] \\
& \text { vi) }(a, c, A) B \rightarrow A(a, c, B) \\
& \text { vii) } \begin{array}{l}
\Uparrow(p, b, R, A) \in \delta(q, a) \\
\quad[q, a, c] \# \rightarrow \bar{A} c \# \text { for } p \in F
\end{array} \\
& \text { viii) } \quad(a, c, A) \bar{B} \rightarrow \vec{A} B \hat{c} \\
& \text { ix) } \# A \rightarrow \# A \\
& \text { x) } \hat{C} A \rightarrow A \hat{c} \\
& \text { xi) } \quad \hat{c} e \rightarrow c e
\end{aligned}
$$


Her er $a, b, d \in T, c, e \in \Sigma$ og $A, B \in \Delta$.

At vi med $L(G)=\# L$ har $L=D$ er en direkte konsekvens af lemma 4.9 og 4.11 samt den bemærkning at produktionerne $x$ ) og $x i)$ sikrer os,at alle elementer $i \sum$ "flyttes helt hen til hojre", inden de konverteres til terminale symboler.

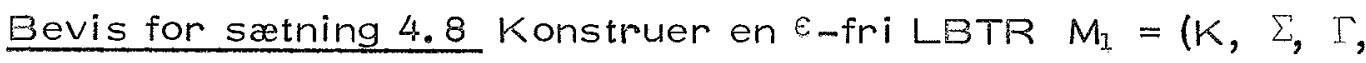
$\left.\Delta \cup\{\Lambda\}, \hat{o}_{1}, q_{0}, F\right)$ ved at sætte

$$
\begin{aligned}
& \prod_{(p, b, x, \Lambda)}^{(p, b} \in \delta_{1}(q, a) \\
& (p, x, \varepsilon) \in \delta(q, a)
\end{aligned}
$$

Iflg. Iemma 4. $13 \mathrm{er}$

$$
D=\{w y \mid(w, y) \in \operatorname{Gr}(M)\}
$$

context sensitivt. Davi uden indskrænkning kan antage, at $\Delta \cap \Sigma=\phi$, kan vi definere homomorfierne $h_{1}, h_{2}$ ved

$$
\begin{aligned}
& h_{I}(\Lambda)=h_{2}(\Lambda)=\epsilon \\
& h_{I}(c)=\left\{\begin{array}{l}
c \text { hvis } c \in \Sigma \\
\varepsilon \text { ellers }
\end{array} \in \Sigma\right. \\
& h_{2}(c)=\left\{\begin{array}{l}
\epsilon \text { hvis } c \\
c \text { ellers }
\end{array}\right.
\end{aligned}
$$

hvorefter det er klart, at

$$
\operatorname{Gr}(M)=\left\{\left(h_{1}(w), h_{2}(w)\right) \mid w \in D\right\}
$$

1 forlængelse af resultaterne fra afsnit 3 er det nu rimeligt at sporge, om sætning 4.8 også gælder "den anden vej", dvs. orn der for et givet $L \in C S$ og to giv ne homomorfier $h_{1}$, $h_{2}$ findes en LBTR $M$ således, at $\operatorname{Gr}(M)=\left\{\left(h_{1}(w), h_{2}(w)\right) \mid w \in L\right\}$. 
Ved hjælp af lemma 2.3 kan vi straks sige, at dette ikke gælder i almindelighed. Lad nemlig $L$ være et sprog, som ikke er context sensitivt og konstruer $L_{1}$ som i lemma 2.3. Definer homomorfierne $h_{1}$, $h_{2}$ ved

$$
\begin{aligned}
& h_{I}(a)=\left\{\begin{array}{l}
\epsilon \text { hvis } a=\# \\
a \text { ellers }
\end{array}\right. \\
& h_{2}(a)=\left\{\begin{array}{l}
\# \text { hvis } a=\# \\
\epsilon \text { ellers }
\end{array}\right.
\end{aligned}
$$

Nus er $h_{1}\left(L_{1}\right)=L \notin C S$, og da Dom $(M) \in C S$ for enhver LBTR $M$ er vi færdige.

Naste spórgsmål er nu, om det er muligt at afgфore for givet $L \in C S$ og givne homomorfier $h_{1}$, $h_{2}$ hvorvidt der findes en LBTR med $\left\{\left(h_{1}(w), h_{2}(w)\right) \mid w \in L\right\}$ som graf.

Svaret gives af $f \phi \mid$ gende

Satning 4. 14 Spprgsmålet om der for givet $L \in C S$ og givne homomorfier $h_{1}$, $h_{2}$ findes en LBTR $M$ med

$$
\operatorname{Gr}(M)=\left\{\left(h_{1}(w), h_{2}(w)\right) \mid w \in L\right\}
$$

er rekursivt uloseligt.

Bevis Lad $L \subseteq \Sigma^{*}$ vare context sensitivt, og lad os betragte det specielle tilfælde, hvor $h_{\mathfrak{Z}}(a)=\epsilon$ for alle $a \in \Sigma$. Der gælder da, at der findes en LBTR $M$ med de onskede egenskaber hvis og kun hvis

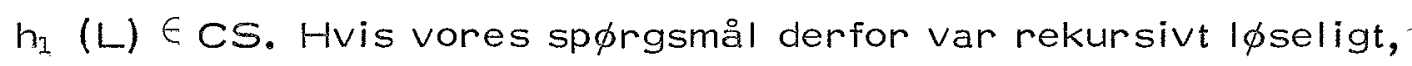
ville det vare rekursivt $\mid \phi s e l i g t$, om det homomorfe billede af et context sensitivt sprog igen er context sensitivt. Iflg. lemma 2.3 implicerer det imidlertid, at det er rekursivt $\mid \phi$ seligt, omet givet Turing sprog er context sensitivt. Beviset f $\phi$ lger herefter af lemma 4. 15. 
Lemma 4.15 Spфrgsmålet, om et givet Turing sprog er context sensitivt, er rekursivt ulфseligt.

Bevis $\operatorname{Lad} T=\left\{x_{1}, \ldots, x_{k}\right\}, U=\left\{w_{1}, \ldots, w_{k}\right\}$ vare PCP-lister over alfabetet $\Sigma$, og lad $G=\left(V_{N}, \Sigma, P, S\right)$ være en grammatik, således at $\varepsilon \notin L(G)$ og $L(G) \notin C S$. Betragt f $\phi$ lgende grammatik $G_{1}=$ $\left(\left\{S_{1}, A, B, C\right\}, \Sigma \cup\{\#\}, P_{1}, S_{1}\right)$ hvor $P_{1}$ er produktionerne

$$
\begin{array}{ll}
S_{1} \rightarrow \# A & \\
A \rightarrow a A a \quad \text { for alle } a \in \Sigma & \\
A \rightarrow B & \\
W_{i} B \times_{i}^{R} \rightarrow B & \\
\# B \# \rightarrow C & \\
C \rightarrow a C \quad \text { for alle a } \in \Sigma \\
C \rightarrow a
\end{array}
$$

$\left(\right.$ Hvis $x=a_{1} \ldots a_{n}$ er $\left.x^{R}=a_{n} \ldots a_{1}\right)$.

Lad endelig Gi være grammatikken $G i=\left(\left\{S_{1}, S, A, B, C\right\} \cup V_{N}\right.$, $\Sigma \cup\{\#\}, P_{1} \cup P_{2} \cup\left\{S i \rightarrow S, S i \rightarrow S_{1}\right\}$, Sii). Det er klart, at $L(G i)=L(G) \cup L\left(G_{1}\right)$. Af konstruktionen af $G_{1}$ folger der nu, at

$$
L\left(G_{1}\right)=\left\{\begin{array}{l}
\phi \text { hvis } \operatorname{PCP}(T, U)=0 \\
\Sigma^{H}-\operatorname{PCP}(T, U)=1
\end{array}\right.
$$

men det betyder åbenbart, at

$$
\begin{aligned}
& \qquad(G I) \text { er context sensitivt } \\
& P C P(T, U)=1
\end{aligned}
$$

Det eneste, $v i$ mangler at vise, er at vi altid effektivt kan konstruere en grammatik $G$, således at $L(G)$ ikke er context sensitiv.

Lad $\left\{\varphi_{x}\right\}$ betegne en nummerering af de partielle rekursive funktioner og lad a vare et element $i \Sigma$. Sproget

$$
L=\left\{a^{n} \mid n \geq 1, \varphi_{n}(n) \text { konvergent }\right\}
$$

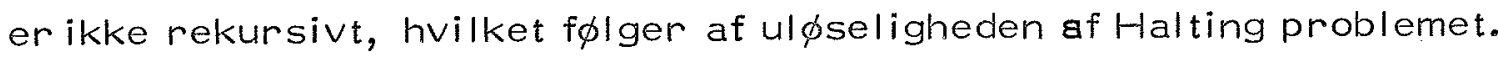


På den anden side er det simpelt at konstruere en Turing Acceptor, der accepterer $L$, det er nemlig den universelle Turing Maskine, der givet $n$ udregner $\varphi(n)$ og accepterer hvis og kun hvis den standser. Givet vor Turing Acceptor $T$ kan vi nu anvende den algoritme, der findes i Hopcroft og Ullimann [1969] til konstruktion af en grammatik for det sprog, der accepteres af $T$.

Lad os ligesom i afsnit 3 slutte dette afsnit med at karakterisere oversættelser defineret af LBTRler ved hjælp af grammatikker.

Definition 4.16 En context sensitiv grammatik med regulær output (CSRO) er et system $G=\left(V_{N_{1}}, V_{N_{2}}, V_{T_{1}}, V_{T_{2}}, P,(S, S i)\right)$ hvor

i) $V_{N_{I}}, V_{N_{2}}$ er alfabeter af hjælpesymboler,

$$
\begin{array}{ll}
V_{T_{1}}, V_{T_{2}}- & - \text { terminale symboler } \\
\operatorname{med} V_{N_{i}} \cap V_{T_{i}}=\phi \quad i=1,2 .
\end{array}
$$

ii) $\left(S\right.$, si) $\in V_{N_{1}} \times V_{N_{2}}$ er startsymbolet

iii) $P$ er en mangde af produktioner af en af folgende former

$$
\begin{aligned}
(\alpha, A) & \rightarrow(\beta, x B) \\
(\alpha, A) & \rightarrow(\beta, x) \\
\text { hvor } \alpha ; \beta & \in V_{1}^{+}|\beta| \geq|\alpha|, A, B \in V_{N_{2}}, x \in V_{T_{2}}^{*}
\end{aligned}
$$

En form er et elenent $(\alpha, x)$ hvor $\alpha \in V_{1}^{+}, x \in V_{2}^{*}$, Definer relationen $\Rightarrow$ mellem former ved

$$
(\alpha, \times A) \Rightarrow(\beta, \eta)
$$

hvis vi kan skrive $(\alpha, x A)$ på formen $(\omega \delta \gamma, x A)$ og $(\beta, \eta)$ på formen $(\omega \mu \gamma, x U) \circ g(\delta, A) \rightarrow(\mu, U)$ er en produktion. 
Med $\stackrel{*}{\Rightarrow}$ som den reflexive, transitive lukning af $\Rightarrow$, definerer $v i$ sproget genereret af $G$ som mangden

$$
L(G)=\left\{(x, y) \in V_{T_{I}^{*}}^{*} V_{T_{2}^{*}}^{*} \mid(S, s i) \stackrel{*}{\Rightarrow}(x, y)\right\}
$$

Ovenstående er et specialtilfælde af Culik llis og Moreyis [1971] definition af binær grammatik og af det sprog, der $\alpha$-genereres af en sådan.

Vi har folgende sæetning fra samme artikel.

Satning 4. 17 Lad $\Sigma, \Delta$ vare to alfabeter og lad $T \subseteq \Sigma^{*} \times \Delta^{*}$. Der findes da en LBTR $M$ med $\operatorname{Gr}(M)=T$ hvis og kun hvis der findes en CSRO $G$ med $L(G)=T$.

Bevis Beviset er gennemf $\phi r t i$ alle pinlige detaljer $i$ ovenstående artikel, hvorfor vi ikke skal komme nфjere ind på det. Ideen er meget kort den, at vi givet en LBTR $M$ med $T=G r(M)$ konstruerer en CSRO, hvis context sensitive del praktisk taget er den samme som grammatikken i lemma 4.9 og hvis regul are del kun indeholder et hjælpesymbol og tre forskellige produktioner $S \rightarrow S, S \rightarrow y s, S \rightarrow \epsilon$ hvor $y$ er en output streng fra $M$.

En produktion, der "stammer" fra en bevægelse, hvor automaten giver output $y$, har formen $(\alpha \rightarrow \beta, S \rightarrow y S)$, en "hjælpeproduktion" er af formen $(\alpha \rightarrow \beta, S \rightarrow S)$ og endelig er den sidst anvendte produktion $i$ en derivation produktionen $(\alpha \rightarrow \beta, S \rightarrow \varepsilon)$.

Har vi omvendt givet en CSRO konstruerer vi en LBTR, der accepterer sproget genereret af den context sensitive del af grammatikken v.hj.a. en "top-down analyse" (jfr. Hopcroft og Ullimann [1969] Theorem 8. 1) og som samtidig anvender den regulære del af grammatikken til at generere det korrekte output.

$\checkmark i$ skal ikke her komme yderligere und på oversattelser defineret af LBTRler, men slutter med at henvise til Culik II og Morey, hvor specielt sammenhængen mellem oversættelser og binære grammatikker er behandlet $i$ dybden. 


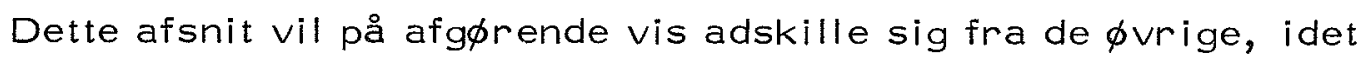
vi her vil bruge en helt anden type argumenter. Medens de hidtige beviser har været formelle og stringente $i$ den forstand, at vi $f$. eks. har konstrueret grammatikker og automater $i$ alle tekniske detaljer, vil vi nu slå ind på "de store armbevægelsers metode", eller hvad der er en mere korrekt betegnelse, vi vil anvende Church's Thesis. Denne siger som bekendt, at en Turing maskine kan gфre alt det, som vi kan give en effektiv beskrivelse af. Derfor vil beviser $\mathrm{i} h \phi j$ grad blive givet $\mathrm{i}$ form af flow-charts, hvis elementer beskriver en beregning, som intuitivt er effektiv.

F申rst en formel definition.

Definition 5.1 En Turing acceptor (TA) er et system $M=(K, \Sigma$, $\left.\Gamma, \delta, q_{0}, F\right)$ hvor $K, \Sigma, \Gamma, \delta, q_{0}$, F har samme betydning som $i$ def. $4.1 \mathrm{og}$ der yderligere gælder, at der findes et specielt symbol $B \in I \backslash \Sigma-B$ er den blanke -.

Ind f $\phi r$ relationen $L^{*}$ pa $\Gamma^{*} K \Gamma_{*}^{*}$ ved for $u, v \in \Gamma^{*}, c \in \Gamma$ at sætte

$$
\begin{aligned}
& \text { i) } \rrbracket_{\delta(p, a) \ni(q, b, N)}^{\text {upav } 1-\text { uqbv }} \quad \prod_{\delta(p, B) \ni(q, b, N)}^{u p \mid-u q b}
\end{aligned}
$$

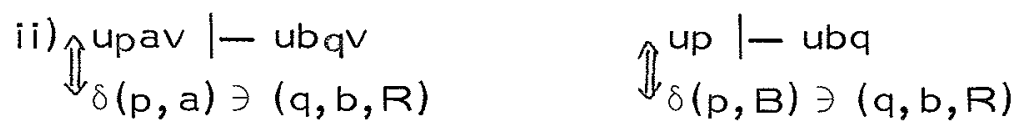

$$
\begin{aligned}
& \text { iii) } \left.\begin{array}{l}
\text { ucpav } 1-\text { uqcbv } \\
\text { pav } 1-\text { - } B a v
\end{array}\right\} \Longleftrightarrow \delta(p, a) \ni(q, b, L) \\
& \left.\begin{array}{l}
\text { ucp } \mid- \text { uqcb } \\
p \mid-q B b
\end{array}\right\} \Longleftrightarrow \delta(p, B) \ni(q, b, L)
\end{aligned}
$$


Med 1 * som den reflexive, transitive lukning af $\mid$ - er sproget accepteret af $M$ mængden

$$
T(M)=\left\{w\left|\exists q \in F, \alpha, \beta \in \Gamma^{*}: q_{0} w\right|^{*} \alpha q \beta\right\}
$$

Det $f \phi$ lger a $f$ definitionen, at hvis $q \in F$ kan $v i$ uden indskrænkning antage, at $M$ standser, dvs. at $\delta(q, a)$ er udefineret for alle $a \in \Gamma$.

Det er velkendt, at de mere udbyggede modeller af TA'er, dvs. maskiner med flere tapes af flere dimensioner og med flere læseskrive hoveder, alle er ækvivalente med ovenstående model.

På basis af def. 5. 1 kan vi nu definere begrebet en Turing oversætter (TTR) ved at tilf $\phi j e$ et outputalfabet $\Delta$, pille lidt ved definitionerne af $\delta$ og $\mid$, og dermed nå frem til at definere grafen for en $\operatorname{TTR} M=\left(K, \Sigma, \Gamma, \Delta, \delta, q_{0}, F\right)$ som

$$
\begin{aligned}
\operatorname{Gr}(M)=\left\{(x, y) \in \Sigma^{*} x \Delta^{*} \mid\right. & \exists \mathrm{q} \in F, \alpha, \beta \in \Gamma^{*}: \\
& \left.\left(q_{0}, w, \varepsilon\right) \mid \stackrel{*}{*}(\alpha q \beta, \varepsilon, y)\right\}
\end{aligned}
$$

De ovenfor beskrevne skridt er fuldstandig parallelle till def. 4.3 og def. 4.4, og det skulle være indlysende, hvordan det går.

Lad os nu vise folgende parallel til corollar 3. 11.

Sætning 5.2 Lad $\Sigma, \Delta$ være to alfabeter med $\Sigma \cap \Delta=\varnothing$ og lad $T \subseteq \Sigma^{*} X \Delta^{*}$. Der findes da en TTR $M$ med $T=\operatorname{Gr}(M)$ hvis og kun hvis der findes et Turing sprog $L$ og to homomorfier $h_{1}, h_{2}$ således at

$$
T=\left\{\left(h_{1}(w), h_{2}(w)\right) \mid w \in L\right\}
$$

Bevis $L$ ad $M=\left(K, \Sigma, I, \Delta, \delta, q_{0}, F\right)$ være en $T T R$ med $T=$ $\operatorname{Gr}(M)$. Konstruer en TA $M_{1}$ på f $\phi$ lgende måde. $M_{1}$ har tre tapes, inputalfabet $\Sigma \cup \Delta$ og virker i overensstemmelse med f $\phi \mid$ gende diagram idet tape 1 bruges som inputtape. 
5.3

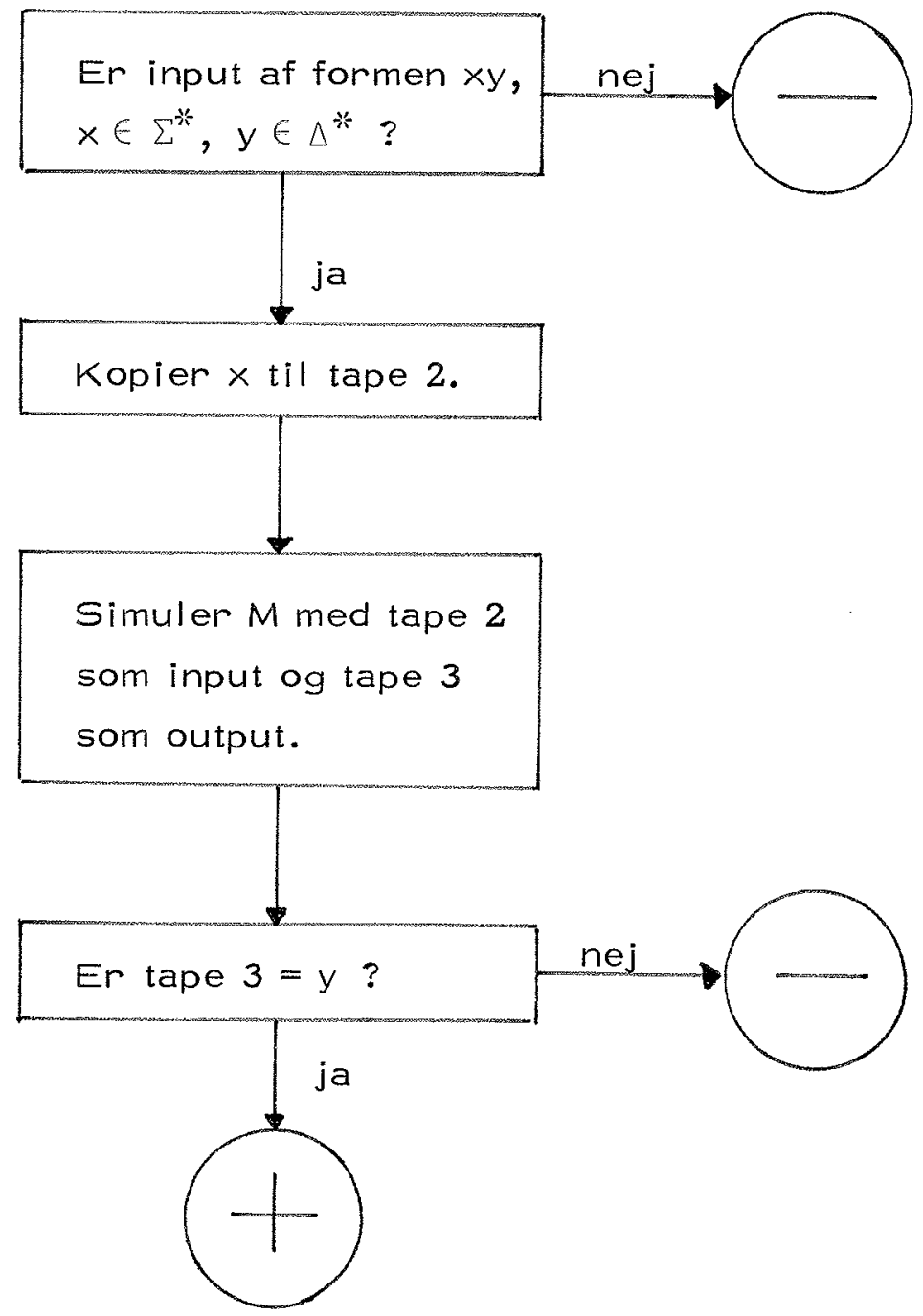

Bemærk at det er muligt, at vor maskine aldrig bliver færdig med at simulere M. (-) angiver en ikke-accepttilstand., medens ( + angiver en accepttilstand.

Lad $L$ være lig med $T\left(M_{1}\right)$ og definer homomorfierne $h_{1}$ og $h_{2}$ ved

$$
\begin{aligned}
& \text { for } a \in \sum \text { er } h_{1}(a)=a, h_{2}(a)=e \\
& \text { for a } \in \Delta \text { er } h_{1}(a)=\varepsilon, h_{2}(a)=a
\end{aligned}
$$

Det er klart at $T=\left\{\left(h_{1}(w), h_{2}(w)\right) \mid w \in L\right\}$

Lad omvendt $T=\left\{\left(h_{1}(w), h_{2}(w)\right) \mid w \in L\right\}$, hvor $L \in R E$ med $L \subseteq \Sigma 1^{*}$. Vi anvender atter lemma 2.3 til at opnå $L^{\prime} \in C S$, $L^{\prime} \subseteq\left(\Sigma^{\prime} \cup\{\#\}\right)^{*}, h\left(L^{\prime}\right)=L$, hvor homomorfien $h$ er defineret 
ved $h(\#)=e, h(a)=a$ for $a \in \sum$. Vi har nu at

$$
T=\left\{\left(h_{1} h(w), h_{z} h(w)\right) \mid w \in L^{\prime}\right\}
$$

Lad $\left\{x_{i}\right\}$ være en nummerering af strengene i $\left(\Sigma^{\prime} \cup\{\#\}\right)^{*}$ og konstruer f $\phi$ lgende TTR med inputalfabet $\Sigma$, outputalfabet $\Delta$ og 4 tapes med tape 1 som input.

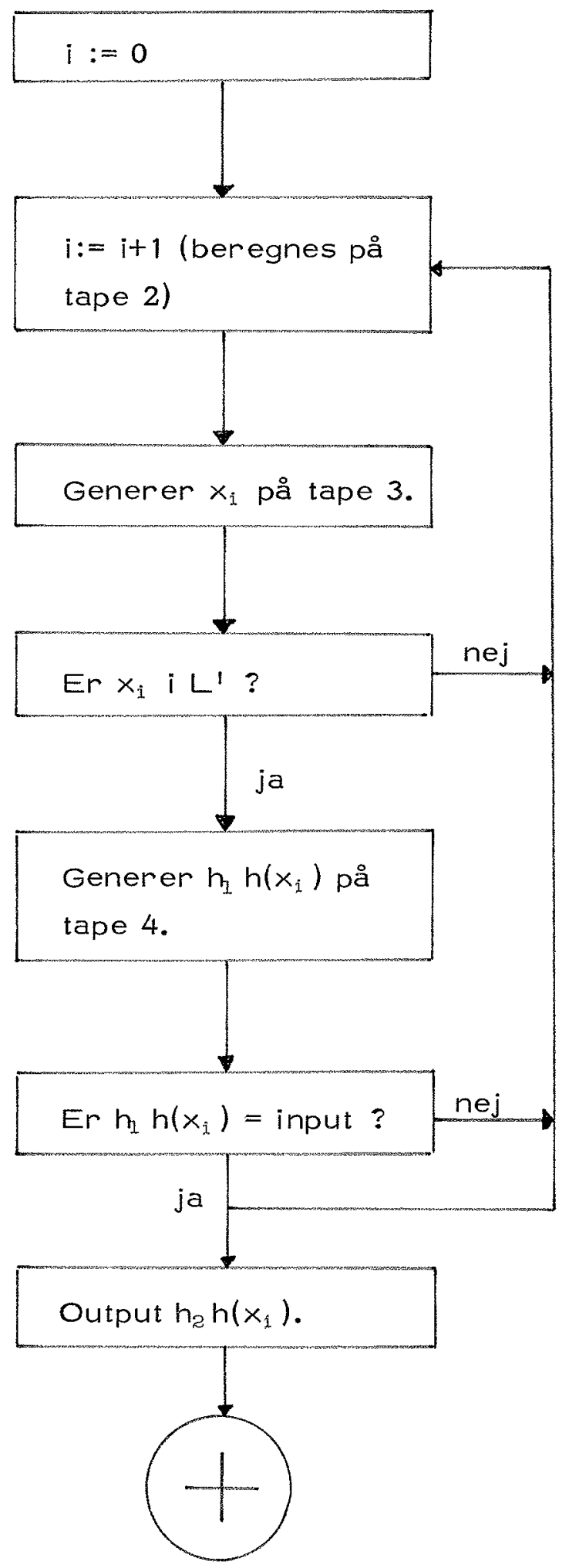


Bemærk at det efter svaret ja i næstsidste kasse er to muligheder for TTR'en. Dette skyldes, at vi må bevare muligheden for at få flere forskellige output fra samme input. Den ekstra detalje med at indf $\phi r e L^{\prime} \in$ CS skyldes, at vi i kasse nr. 4 skal have et spфrgsmål, som er rekursivt $\mid \phi$ seligt.

Lad os som kommentar til ovenstående sætning anfore, at den også kunne have været vist på folgende måde. Turing sprogene er en full AFL $J$, hvorfor der iflg. sætning 2.6 findes en AFTR D så $\mathcal{L}(\mathcal{D})=\mathcal{J}$. Anvend sætning 3.6 på $\mathscr{D}$ og vi er tilsyneladende hjemme. Her er imidlertid en ting at være opmærksom på, og det er kravet om, at vore automater i en AFTR er envejs au tomater. Ovenstående klasse $\mathcal{D}$ er altså en klasse af envejsautomater, som ganske vist er ækvivalent med klassen af Turing maskiner $i$ den forstand, at de accepterer de samme sprog, men som strukturelt set ikke behфver at ligne de klassiske Turing maskiner. Dette er grunden til, at vi har anvendt den mere direkte måde at angribe problemet på.

Lad os igen slutte med at angive sammenhængen mellem oversættelser og grammatikker. Ved at ændre definitionen af en context sensitiv grammatik med regulaer output (CSRO i def. 4. 16) således at vi $i$ en produktion $\left(\alpha, A_{1}\right) \rightarrow(\beta, \times B)$ eller $(\alpha, A) \rightarrow(\beta, x)$ ikke længere kræver at $|\beta| \geq|\alpha|$ făr vi, hvad vi kan kalde en Turing grammatik med regulær output (RERO). Det er ikke svært at vise folgende sætning, hvormed vi slutter behandlingen af TTR'er.

Sætning 5.3 Lad $\Sigma, \Delta$ være to alfabeter og lad $T \subseteq \Sigma^{*} \times \Delta^{*}$. Der findes da en TTR $M$ med $G r(M)=T$ hvis og kun hvis der findes en RERO $G$ med $T=L(G)$. 
Hvis vi anvender de sædvanlige typebetegnelser for Chomskyhierarkiet - dvs. et type 1 sprog er context sensitivt, en type 2 automat er en PDA osv. - har vi i de foregående afsnit vist, at givet en oversætter $M$ af type $i$, findes der et sprog $L$ af type $i$ og to homomorfier $h_{1}, h_{2}$ således at

$$
\operatorname{Gr}(M)=\left\{\left(h_{1}(w), h_{2}(w)\right) \mid w \in L\right\}
$$

Bortset fra tilfældet $i=1$ har vi vist at denne sætning også gælder "den anden vej" ligesom vi har demonstreret at i tilfældet $i=1$ er det rekursivt ul $\phi$ seligt, hvorvidt dette sidste er opfyldt. Endelig har vi i hvert tilfælde angivet en karakterisation for graferne af oversætterne v.hj.a. "par af grammatikker".

I spфrgsmålet om "anvendeligheden" af den præsenterede teori er det nok forst og fremmest push-down oversætterrie der kommer i betragtning. Lad os prove at anvende en af vore sætninger på et problem, der optræder i forbindelse med syntaxdirigeret oversættelse. Aho og Ullman [1971] anf $\phi$ rer et eksempel som $i$ alt væsentligt er f $\phi \mid$ gende:

Betragt en PASCAL-lignende produktion som

$<$ for statement $::=$ for $<$ assignmemt $>$ to $<$ integer $>$ do $<$ statement $>$

Et eksempel på et <for statement $>$ kan være

$$
\text { for } 1 \leftarrow J+1 \text { to } N \text { do } B \leftarrow B+A[J]
$$

En naturlig kode at producere vil være

1. Udf $\phi r$ assignmentet

2. Test om $\mathrm{I} \leq \mathrm{N}$

3. Hvis ikke hoppes der ud 


\section{Uldf $\phi r$ statementet \\ 5. Sæt $1 \leftarrow 1+1$ \\ 6. Gå tilbage til 2}

Vi kan nu sp $\phi r g e$, om denne oversættelse kan udf $\phi$ res af en PDTR. En nфdvendig og tilstrækkelig betingelse herfor er iflg. sætning 3. 13 at den oversatte kode kan beskrives af en produktion af formen

$<$ for statement $>::=t_{1}<$ assignment $>t_{2}<$ integer $>t_{3}<$ statement $>t_{4}$

hvor $t_{1}$ for $1 \leq i \leq 4$ er terminale strenge.

Vi kan imidlertid med det samme se, at vi i vor kode făr brug for to "oversættelser" af <assignment>, nemlig dels den kode, der udf $\phi$ rer $1 \leftarrow J+1$, og dels en kode, der kan levere l's reference, som vi skal bruge i 2 og 5. Da <assignment> i (*) imidlertid kun kan have en "værdi", kan ovenstående oversættelse ikke defineres af en PDTR.

Lad os prove at omskrive produktionen til

$\begin{aligned}<\text { for statement }:: & =\frac{\text { for }}{<\text { identifier }>r<\text { integer expression }>\text { to }} \\ & <\text { integer }>\text { do }<\text { statement }\end{aligned}$

Lad os vise at vi nu faktisk kan definere oversættelsen v.hj. a. en PDTR ved at anf $\phi r e$ en oversættelse til GIER' s assemblerkode SLIP. Vi skal altså vise, at vi kan oversætte strengen

$$
\text { for } 1 \leftarrow E \text { to } N \text { do } S
$$

Lad os antage, at den semantiske værdi af I er en stump kode, der anbringer adressen på den aktuelle identifier i R-registerets adressedel. Den semantiske værdi af $E$ hhv. $N$ er den kode, der udregner den aktuelle værdi af $E$ hhv. Nog anbringer denne $i R$ og endelig er den semantiske værdi af $S$ den kode, der udregner $\mathbf{S}$. 
Vi kan nu skrive vor semantiske produktion af formen

$$
\text { <for statement>::= } t_{1} \mid t_{2} E t_{3} N t_{4} S t_{5}
$$

på f $\phi \mid$ gende måde

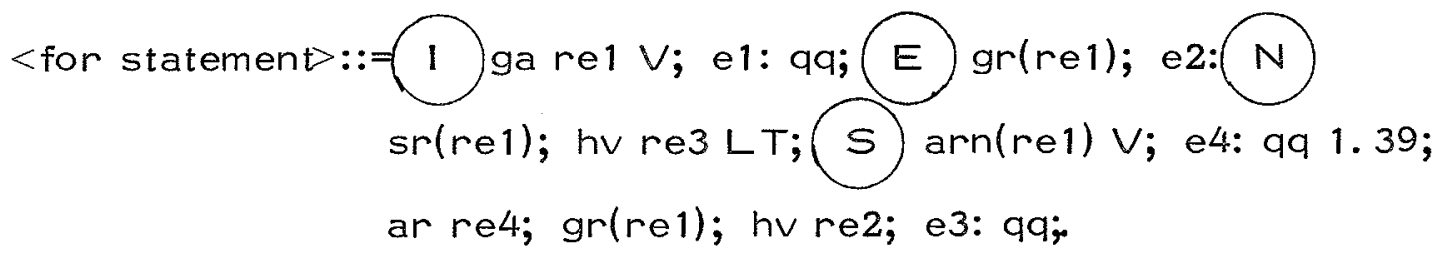

hvor vi har brugt; som skilletegn mellem SLIP-instruktioner.

Et andet spфrgsmål, der melder sig i forbindelse med praktiske anvendelser, er spфrgsmålet om at karakterisere de oversættelser, der defineres af deterministiske push-down automater.

En SSDTS $G=(V, \Sigma, \Delta, R, S)$ (jfr. def. 3. 12) kaldes omvendt polsk, såfremt der for enhver form $(\alpha, \beta)$ gælder at $\beta \in V^{*} \Sigma^{*}$. $G$ siges at være $L R(k)$ såfremt grammatikken $G^{\prime}=(V, \Sigma, P, S)$ er en LR(k)-grammatik. Her er $P$ produktionerne $A \rightarrow \alpha$, hvor $A \rightarrow(\alpha, \beta)$ er en regel i $R$.

Lewis og Stearns [1968] indeholder nu f $\phi$ lgende resultat.

Satning 6.1 Lad $\Sigma, \Delta$ være to alfabeter og lad $T \subseteq \Sigma^{*} X \Delta^{*}$. Der findes da en deterministisk push-down oversætter $M \operatorname{med} G r(M)=$ $T$ hvis og kun hvis der findes en omvendt polsk LR(K) SSDTS $G=(V, \Sigma, \Delta, R, S)$ således at $L(G)=T$.

Beviset for sætning 6.1 er konstruktivt, og sætningen har folgelig anvendelser $i$ forbindelse med konstruktion af $L R(k)$-parsere og $L R(k)$-parser-generatorer.

Lad os alutte med at anfore, at der ikke findes nogen "homomorfikarakterisation" af oversættelser defineret af deterministiske PDAler. Et fors $\phi g$ på at g $\phi$ re det vil utvivlsomt medf $\phi$ re overvejelser af samme art som for LBA'erne $i$ afsnit 4 . Det springende punkt er igen, at deterministiske sprog ikke er lukket over for homomorfi. 


\section{Referencer:}

Aho and Ullman [1969]: "Syntax Directed Translations and the Pushdown Assembler". J. Comp. Syst. Sci. 3, (37-56)

Aho and Ullman [1969a]: "Properties of Syntax Directed Translations." J. Comp. Syst. Sci. 3, (319-334)

Aho and Ull man [1971]: "Translations on a Context Free Grammar." Inf. and Control 19, (439-475)

Culik II and Morey [1971]: "Formal Schemes for Language Translations." Intern. J. Computer Math. A, 3, (17-48)

Ginsburg and Greibach [1969]: "Abstract Families of Languages." Mem. of the Amer. Math. Soc., 87, (1-32)

Ginsburg and Rose [1966]: "Preservation of Languages by Transducers." Inf. and Control, 9, (153-176)

Ginsburg and Rose [1968]: "A note on perservation of Languages by Transducers. "Inf. and Control, 12, (549-552)

Hopcroft and Ullman [1969]: "Formal Languages and their Relation to Automata." Addison-Wesley Inc.

Ibarra [1971]: "Characterizations of Transductions Defined by Abstract Families of Transducers." J. Math. Syst. Th. 5, 3, (271-281)

Kuroda [1964]: "Classes of Languages and Linear-Bounded Automata." Inf. and Control (207-223)

Landweber [1963]: "Three Theorems on Phrase Structure Grammars of Type 1."Inf. and Control (131-136)

Lewis and Stearns [1968]: "Syntax-Directed Transduction." JACM, $15,3(465-468)$ 